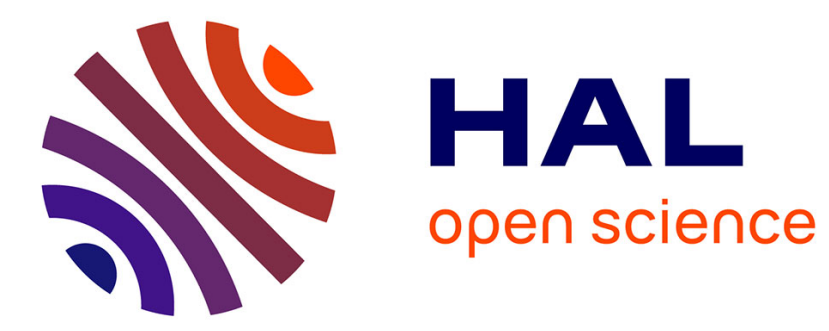

\title{
Exemples de comptage de courbes sur les surfaces
}

David Bourqui

\section{To cite this version:}

David Bourqui. Exemples de comptage de courbes sur les surfaces. Mathematische Annalen, 2013, 357 (4), pp.1291-1327. 10.1007/s00208-013-0933-2 . hal-00697614

\section{HAL Id: hal-00697614 https://hal.science/hal-00697614}

Submitted on 15 May 2012

HAL is a multi-disciplinary open access archive for the deposit and dissemination of scientific research documents, whether they are published or not. The documents may come from teaching and research institutions in France or abroad, or from public or private research centers.
L'archive ouverte pluridisciplinaire HAL, est destinée au dépôt et à la diffusion de documents scientifiques de niveau recherche, publiés ou non, émanant des établissements d'enseignement et de recherche français ou étrangers, des laboratoires publics ou privés. 


\title{
EXEMPLES DE COMPTAGES DE COURBES SUR LES SURFACES
}

\author{
par
}

David Bourqui

\begin{abstract}
Résumé. - Soit $X$ une surface dont l'anneau de Cox a une seule relation, laquelle vérifie en outre une certaine propriété de linéarité. Nous montrons, sous une hypothèse simple, que les conjectures de Manin géométriques valent pour certains degrés du cône effectif dual de $X$ (notamment pour ces degrés l'espace de modules de morphismes a la dimension attendue). Le résultat s'applique à une classe de surfaces de del Pezzo généralisées qui a été intensément étudiée dans le cadre de la conjecture de Manin arithmétique.
\end{abstract}

Some examples of curves countings on surfaces. Let $X$ be a surface whose Cox ring has a single relation satisfying moreover a kind of linearity property. Under a simple assumption, we show that the geometric Manin's conjectures hold for some degrees lying in the dual of the effective cone of $X$ (in particular, for those degrees the moduli space of morphisms has the expected dimension). The result applies to a class of generalized del Pezzo surfaces which has been intensively studied in the context of the arithmetic Manin's conjecture.

MSC : 11G50, 14J26, 14C20, 14D22, 14H10

\section{Introduction}

Dans ce texte, nous poursuivons notre investigation de la conjecture de Manin sur le comportement asymptotique du nombre de courbes de degré borné sur les « hypersurfaces intrinsèques linéaires », c'est-à-dire les variétés dont l'anneau total de coordonnées s'identifie à l'anneau d'une hypersurface affine dont l'équation vérifie en outre une certaine propriété de linéarité. Nous renvoyons à la section 3 pour plus de précisions sur la terminologie. Signalons d'ores et déjà que la classification de Derenthal des surfaces de del Pezzo généralisées de degré $\geqslant 3$ dont l'anneau total de coordonnées n'a qu'une équation (cf. [Der06]) montre que les 21 surfaces qu'il obtient tombent toutes dans la classe considérée, sauf une (l'une des deux surfaces cubiques avec singularité $\mathbf{D}_{4}$ ). Ces surfaces ont été intensément étudiées dans le cadre de la conjecture de Manin arithmétique (évaluation asymptotique du nombre de points 
rationnels de hauteur bornée lorsque le corps de base est le corps des rationnels), $c f$. [BBD07, BB07, DT07, BD09a, Der09, BD09b, Lou10, LB12, LB11]

Le résultat principal obtenu dans cet article est le suivant. Nous l'énonçons dans cette introduction sous une forme volontairement simplifiée.

Théorème 1.1. - Soit $k$ un corps parfait et $\mathscr{C}$ une $k$-courbe projective, lisse et géométriquement intègre de genre $g_{\mathscr{C}}$.

Soit $X$ une surface projective, lisse et géométriquement intègre définie sur $k$ qui est une hypersurface intrinsèque linéaire, et qui vérifie en outre l'hypothèse 4.4 ci-dessous.

Alors $X$ satisfait une version partielle des conjectures de Manin géométriques.

Le résultat est donné sous sa forme précise par le théorème 6.2. Nous nous contentons ici de quelques commentaires.

Tout d'abord, dans cet article, l'appellation « conjectures de Manin géométriques » recouvrira d'une part un cadre où le corps $k$ est supposé fini et où on s'intéresse à la problématique du comptage asympotique de courbes de degré borné, et d'autre part un cadre où le corps $k$ peut être a priori quelconque, et où on étudie la dimension et le nombre de composantes des espaces de modules de morphismes de courbes de degré donné. La section 2 contient plus de détails sur la forme précise des conjectures étudiées dans ce texte.

Ensuite, la partialité évoquée dans l'énoncé ci-dessus est explicitement quantifiable : le théorème dit en gros que pour les cas considérés, la conjecture de Manin vaut sur une certaine partie explicite du dual $C_{\text {eff }}(X)^{\vee}$ du cône effectif de $X$. Par exemple le résultat dit que sur cette partie, l'espace de modules de morphismes est irréductible et a la dimension attendue.

Pour deux surfaces de la liste de Derenthal (en fait pour trois, mais pour la troisième le résultat était déjà connu d'après nos travaux précédents) on obtient les conjectures de Manin « complètes », c'est-à-dire que les conjectures valent sur $C_{\text {eff }}(X)^{\vee}$, et pour cinq autres on obtient une version « complète à la limite» de ces conjectures (on peut trouver des parties -en fait des réunions de cônes- sur lesquelles les conjectures valent et dont le volume est aussi proche que l'on veut de celui de $\left.C_{\text {eff }}(X)^{\vee}\right)$, cf. le corollaire 6.6. Pour toutes les autres, on peut calculer explicitement la proportion de $C_{\text {eff }}(X)^{\vee}$ sur laquelle notre méthode montre que les conjectures valent, $c f$. la table 1. En particulier, les résultats de [Bou11b] (qui concernaient le cadre de l'étude de la dimension des espaces de modules) sont améliorés. Nous renvoyons à la section 6 pour plus de précisions.

Enfin, il faut souligner que le théorème 6.2 isole le terme principal attendu et identifie explicitement le « terme d'erreur » qui est la cause de la partialité dans l'énoncé ci-dessus. Il montre en que les conjectures complètes sont vérifiées si et seulement si ce dernier terme peut être contrôlé de manière ad hoc. La méthode employée dans ce texte ne nous permet d'obtenir ce contrôle que sur certaines régions du dual du cône effectif, celles où les coefficients de l'équation de l'anneau de coordonnées total de $X$ sont de degré « suffisamment grand ». Si cette dernière condition n'est pas vérifiée, il faut tenir compte des syzygies en bas degré, ce qui semble délicat (mais peut-être pas insurmontable). 
Le schéma général de la stratégie employée dans cet article est le même que pour [Bou09a, Bou11a, Bou11b] : relèvement du problème de comptage en termes de l'anneau total de coordonnées de $X$, inversion de Möbius, comptage de sections globales vérifiant certaines équations linéaires à l'aide de Riemann-Roch et d'algèbre linéaire élémentaire et étude des séries génératrices en découlant.

Cependant, dans le présent travail, l'utilisation d'une inversion de Möbius «partielle » et non plus totale jointe à une étude plus fine des séries génératrices intervenant permettent d'affaiblir et de simplifier de manière significative les hypothèses qui étaient nécessaires dans [Bou09a, Bou11a, Bou11b], dont certaines exigeaient des calculs lourds et peu éclairants pour leur éventuelle vérification, et n'étaient d'ailleurs pas vérifiées pour toutes les surfaces de la liste de Derenthal. La seule hypothèse nécessaire est ici l'hypothèse 4.4 , qui est très simple à vérifier pour peu qu'on ait une description explicite de l'anneau de coordonées total de la variété considérée. On voit ainsi aussitôt qu'elle est satisfaite par les 20 surfaces de la liste de Derenthal. Peut-être pourrait-t-on d'ailleurs se risquer à espérer qu'elle est satisfaite pour toutes les hypersurfaces intrinsèques linéaires. On montre notamment sous cette seule hypothèse que le terme principal de la fonction zêta des hauteurs correspond bien à celui prédit par Peyre ( $c f$. la relation (4.0.50)). Dans [Bou11a], cette propriété nécessitait a priori une vérification calculatoire au cas par cas ( $c f$. la remarque 11 de op.cit.).

Il est à noter que le résultat final est démontré pour les surfaces, mais que beaucoup d'arguments, notamment ceux utilisés pour le calcul du terme principal, sont valables en toute dimension, et sont donnés dans ce cadre. Il est sans doute possible, au prix de complications techniques, d'étendre les résultats obtenus en dimension supérieure. Il faut remarquer que peu d'exemples explicites d'hypersurfaces intrinsèques linéaires en dimension supérieure semblent connus.

\section{Conjectures de Manin géométriques}

Dans tout cet article, $k$ désigne un corps parfait et $\mathscr{C}$ une $k$-courbe projective, lisse et géométriquement intègre, dont on note $g_{\mathscr{C}}$ le genre. Soit $\operatorname{Div} v_{\text {eff }}(\mathscr{C})$ le monoïde des diviseurs effectifs de $\mathscr{C}$ définis sur $k$. On note $\operatorname{Div}_{\text {eff }}(\mathscr{C})_{\leqslant 1}$ le sous-ensemble de $\operatorname{Div}_{\text {eff }}(\mathscr{C})$ constitué des diviseurs dont toutes les multiplicités valent au plus 1 .

Soit $X$ une $k$-variété projective, lisse et géométriquement intègre définie sur $k$. On suppose que son groupe de Picard géométrique est libre de rang fini et déployé, c'està-dire que l'action du groupe de Galois absolu est triviale. On suppose en outre que $-\mathscr{K}_{X}$, la classe du faisceau anticanonique de $X$ dans le groupe de Picard, est située à l'intérieur du cône effectif $C_{\text {eff }}(X)$ de $X$. On suppose par ailleurs que $C_{\text {eff }}(X)$ est finiment engendré.

Pour $U$ ouvert de Zariski non vide de $X$ assez petit et pour tout élément $y$ du dual $\operatorname{Pic}(X)^{\vee}$ du groupe de Picard de $X$ soit $\operatorname{Hom}_{U}(\mathscr{C}, X, y)$ la $k$-variété quasi-projective paramétrisant les morphismes $f: \mathscr{C} \rightarrow X$ dont l'image rencontre $U$ et de degré absolu $\left[f_{*} \mathscr{C}\right]=y$.

Si $k$ est un corps fini de cardinal $q$, on note $h_{\mathscr{C}}$ le nombre de classes de diviseurs de degré 0 et $\mathscr{C}^{(0)}$ l'ensemble des points fermés de $\mathscr{C}$. Pour $v \in \mathscr{C}^{(0)}$, on note $\kappa_{v}$ le 
corps résiduel et $q_{v}=q^{f_{v}}$ son cardinal. On pose alors

$$
\gamma(X) \stackrel{\text { déf }}{=}\left(\frac{h_{\mathscr{C}} q^{\left(1-g_{\mathscr{C}}\right)}}{q-1}\right)^{\mathrm{rg}(\operatorname{Pic}(X))} q^{\left(1-g_{\mathscr{C}}\right) \operatorname{dim}(X)} \prod_{v \in \mathscr{C}(0)}\left(1-q_{v}^{-1}\right)^{\operatorname{rg}(\operatorname{Pic}(X))} \frac{\# X\left(\kappa_{v}\right)}{q_{v}^{\operatorname{dim}(X)}} .
$$

L'interprétation conceptuelle de $\gamma(X)$ se fait en termes du volume de l'espace adélique associé à la variété $X \times_{k} k(\mathscr{C})$ pour une certaine mesure de Tamagawa, $c f$. [Pey95, Pey03]. Sous les seules hypothèses que nous avons données, la convergence du produit eulérien dans (2.0.1) n'est a priori pas assurée. Nous renvoyons à [Pey03] pour des précisions sur les hypothèses permettant de montrer la convergence en utilisant WeilDeligne. Pour la classe de variétés étudiée dans cet article, ces hypothèses sont vérifiées et la convergence du produit peut d'ailleurs se voir de manière élémentaire.

Définition 2.1. - Soit $\left(a_{n}\right) \in \mathbf{C}^{\mathbf{N}}, \rho>0$ et $k \in \mathbf{N}$. On dit que la série $\sum a_{n} t^{n}$ est $\rho$-contrôlée à l'ordre $r$ s'il existe $\left(b_{n}\right) \in \mathbf{R}^{\mathbf{N}}$ telle que $\left|a_{n}\right| \leqslant b_{n}$, le rayon de convergence de $\sum b_{n} t^{n}$ est supérieur à $\rho$ et sa somme se prolonge en une fonction méromorphe sur un disque de rayon strictement supérieur à $\rho$, ayant des pôles d'ordre au plus $r$ sur le cercle de rayon $\rho$.

Voici les versions de la conjecture de Manin géométrique que nous considèrerons dans cet article. Pour nous, un cône sera toujours polyedral rationnel.

Définition 2.2. - Soit $U$ un ouvert non vide de $X$ et $\mathcal{P}$ une partie de $C_{\text {eff }}(X)^{\vee}$ qui est une réunion finie de cônes.

1. On suppose que $k$ est un corps fini. On dit que $(X, U)$ vérifie « Manin I sur $\mathcal{P}$ » si la série

$$
\sum_{y \in \mathcal{P} \cap \operatorname{Pic}(X)^{\vee}}\left(\# \operatorname{Hom}_{U}(\mathscr{C}, X, y)(k)-\gamma(X) q^{\left\langle y,-\mathscr{K}_{X}\right\rangle}\right) t^{\left\langle y,-\mathscr{K}_{X}\right\rangle}
$$

est $q^{-1}$-contrôlée à l'ordre $\operatorname{rg}(\operatorname{Pic}(X))-1$, et on dit que $(X, U)$ vérifie « Manin II sur $\mathcal{P} »$ si on a

$$
\lim _{\substack{y \in \mathcal{P} \cap \operatorname{Pic}(X)^{\vee} \\ \operatorname{dist}(y, \partial \mathcal{P}) \rightarrow+\infty}} q^{\left\langle y, \mathscr{K}_{X}\right\rangle} \# \operatorname{Hom}_{U}(\mathscr{C}, X, y)(k)=\gamma(X) .
$$

On dit que $(X, U)$ vérifie « Manin I fort sur $\mathcal{P} »$ si pour toute partie $\mathcal{P}^{\prime}$ de $\mathcal{P}$ qui est une union finie de cônes, $(X, U)$ vérifie « Manin I sur $\mathcal{P}^{\prime}$ ».

2. On dit que $(X, U)$ vérifie « Manin géométrique sur $\mathcal{P}$ » s'il existe un réel positif $M$ (dépendant de $X, \mathscr{C}$ et $\mathcal{P}$ ) telle que pour tout $y \in C_{\text {eff }}(X)^{\vee} \cap \operatorname{Pic}(X)^{\vee}$ vérifiant $\operatorname{dist}(y, \partial \mathcal{P}) \geqslant M$, la variété $\operatorname{Hom}_{U}(\mathscr{C}, X, y)$ est géométriquement irréductible de dimension $\left\langle y,-\mathscr{K}_{X}\right\rangle+\left(1-g_{\mathscr{C}}\right) \operatorname{dim}(X)$.

Pour $\alpha \in\{I, I$ fort, $I I$, géométrique $\}$, on dit que $(X, U)$ vérifie « Manin $\alpha »$ si $(X, U)$ vérifie « Manin $\alpha$ sur $C_{\text {eff }}(X)^{\vee} »$.

Remarque 2.3. - Notons $\rho(X)=\operatorname{rg}(\operatorname{Pic}(X))$ et, pour toute partie mesurable $\mathcal{P}$ de $C_{\text {eff }}(X)^{\vee}$, notons $\alpha_{\mathcal{P}}(X)$ le volume de l'intersection de $\mathcal{P}$ avec l'hyperplan affine $\left\{\left\langle\bullet,-\mathscr{K}_{X}\right\rangle=1\right\}$ (la mesure étant normalisée par le réseau $\operatorname{Pic}(X)^{\vee}$ ). Soit 
$\delta \stackrel{\text { déf }}{=} \operatorname{Max}\left\{d \in \mathbf{N}_{>0}, \quad \frac{1}{d} \mathscr{K}_{X} \in \operatorname{Pic}(X)\right\}$. Grâce aux estimées de Cauchy, on voit que « Manin I sur $\mathcal{P}$ » équivaut à l'asymptotique suivante pour le nombre de courbes de degré anticanonique borné :

$$
\sum_{\substack{y \in \mathcal{P} \cap \operatorname{Pic}(X)^{\vee} \\\left\langle y,-\mathscr{K}_{X}\right\rangle=\delta d}} \# \operatorname{Hom}_{U}(\mathscr{C}, X, y)(k)=\alpha_{\mathcal{P}}(X) \gamma(X) d^{\rho(X)-1} q^{\delta d}+\underset{\substack{\mathcal{O} \\ d \rightarrow+\infty}}{\mathcal{O}}\left(d^{\rho(X)-2} q^{\delta d}\right)
$$

Ainsi « Manin I» équivaut essentiellement à la conjecture de Manin originelle (notons que si $C_{\text {eff }}(X)$ est engendré par des diviseurs de $X \backslash U$, ce qu'on peut toujours supposer si $U$ est assez petit, on a $\operatorname{Hom}_{U}(\mathscr{C}, X, y)=\varnothing$ si $\left.y \notin C_{\text {eff }}(X)^{\vee}\right)$. « Manin I fort », tout comme « Manin II », expriment une sorte de propriété d'équirépartition des degrés absolus des courbes de degré anticanonique $d$ dans le cône effectif dual.

Notons qu'a priori aucune des deux propriétés « Manin I fort » ou 《 Manin II » n'entraîne l'autre. Cependant dans cet article, les cas que nous en établirons découleront d'une même propriété plus forte satisfaite par les $\# \operatorname{Hom}_{U}(\mathscr{C}, X, y)(k)$ (cf. la remarque 6.1 ).

Remarque 2.4. - Pour Manin « géométrique », il est a priori concevable d'étudier des invariants «motiviques » plus fins que la dimension et le nombre de composantes, comme c'est fait dans $[\mathbf{B o u 0 9 b}]$ pour les variétés toriques.

Rappelons que par une application standard de Weil-Deligne et la théorie élémentaire de la déformation des morphismes (cf. e.g. [Deb01]), on a la propriété suivante (on suppose pour simplifier que $k=\mathbf{Q}$, mais par les arguments habituels ce procédé de réduction aux corps finis peut se réaliser sur un corps de base quelconque).

Lemme 2.5. - $\boldsymbol{H o m}_{U}(\mathscr{C}, X, y)$ est géométriquement irréductible de dimension $\left\langle y,-\mathscr{K}_{X}\right\rangle+\left(1-g_{\mathscr{C}}\right) \operatorname{dim}(X)$ si et seulement si pour presque tout nombre premier $p$ on $a$

$$
\lim _{r \rightarrow+\infty} p^{r\left[\left(g_{\mathscr{C}}-1\right) \operatorname{dim}(X)+\left\langle y, \mathscr{K}_{X}\right\rangle\right]} \# \boldsymbol{H o m}_{U}(\mathscr{C}, X, y)\left(\mathbf{F}_{p^{r}}\right)=1
$$

\section{Anneau de Cox, inversion de Möbius, et relèvement au torseur universel}

On conserve les notations et hypothèses de la section précédente. On suppose en outre que l'anneau total de coordonnées ou anneau de Cox de $X$ (cf. e.g. [Has04]), noté $\operatorname{Cox}(X)$, est de type fini. Soit $\left\{u_{i}\right\}_{i \in \mathfrak{I}}$ une famille finie de sections globales (non constantes) qui engendre $\operatorname{Cox}(X)$. Pour $i \in \mathfrak{I}$, on note $\mathscr{E}_{i}$ le diviseur des zéros de $u_{i}$. Soit $X_{0}$ l'ouvert de $X$ égal au complémentaire de la réunion des diviseurs $\left\{\mathscr{E}_{i}\right\}_{i \in \mathfrak{I}}$.

3.1. Inversion de Möbius. - Soit

$$
\mathcal{C}_{\text {inc }} \stackrel{\text { déf }}{=}\left\{K \subset \mathfrak{I}, \quad \cap_{i \in K} \mathscr{E}_{i} \neq \varnothing\right\}
$$

et

$$
\mathbf{N}_{\text {inc }}^{\mathfrak{I}}=\left\{\boldsymbol{e} \in \mathbf{N}^{\mathfrak{I}}, \quad\left\{i \in \mathfrak{I}, \quad e_{i} \geqslant 1\right\} \in \mathcal{C}_{\text {inc }}\right\} .
$$


On a la généralisation classique suivante de la formule d'inversion de Möbius ( $c f . e . g$.

[Bou09a, proposition 1.21]).

Proposition 3.1. - Il existe une unique fonction $\mu_{X}^{\circ}: \mathbf{N}^{\mathfrak{I}} \longrightarrow \mathbf{C}$ vérifiant

$$
\forall \boldsymbol{e} \in \mathbf{N}^{\mathfrak{I}}, \quad \sum_{0 \leqslant e^{\prime} \leqslant e} \mu_{X}^{\circ}\left(\boldsymbol{e}^{\prime}\right)=\mathbf{1}_{\mathbf{N}_{\text {inc }}^{\mathfrak{⿰}}} .
$$

En particulier, on a $\mu_{X}^{\circ}(\boldsymbol{\alpha})=0$ dans les cas suivants :

1. il existe $i \in \mathfrak{I}$ tel que $\alpha_{i} \geqslant 2$;

2. $\alpha$ est non nul et l'intersection $\cap_{i, \alpha_{i} \neq 0} \mathscr{E}_{i}$ est non vide; ceci vaut en particulier si on $a \sum_{i \in \mathfrak{I}} \alpha_{i}=1$.

Soit $J$ une partie de $\mathfrak{I}$ et $I=\mathfrak{I} \backslash J$. Pour $\boldsymbol{g} \in \mathbf{N}^{J}, \boldsymbol{f} \in \mathbf{N}^{I}$ on pose

$$
\nu_{X, J}^{\circ}(\boldsymbol{g}, \boldsymbol{f}) \stackrel{\text { déf }}{=} \sum_{0 \leqslant f^{\prime} \leqslant f} \mu_{X}^{\circ}\left(\boldsymbol{g}, \boldsymbol{f}^{\prime}\right) .
$$

Remarque 3.2. - $\mathrm{Si}(0, \boldsymbol{f}) \notin \mathbf{N}_{\mathrm{inc}}^{\mathfrak{I}}$, alors $\nu_{X, J}^{\circ}(\boldsymbol{g}, \boldsymbol{f})$ est nul pour tout $\boldsymbol{g}$.

Remarque 3.3. - Pour $K \subset I$, soit $\boldsymbol{f}_{K} \in\{0,1\}^{I}$ l'élément caractérisé par $f_{K, i}=1$ si et seulement si $i \in K$.

Soit $K \subset I$ tel qu'on ait, pour tout $j \in J,\{j\} \cup K \notin \mathcal{C}_{\text {inc }}$. Alors pour toute partie non vide $J_{1}$ de $J$ et tout $\boldsymbol{g}_{2} \in\{0,1\}^{J \backslash J_{1}}$ on a la relation

$$
\sum_{\boldsymbol{g} \in\{0,1\}^{J_{1}}} \nu_{X, J}^{\circ}\left(\boldsymbol{g}_{1}, \boldsymbol{g}_{2}, \boldsymbol{f}_{K}\right)=0 .
$$

Ceci découle des définitions de $\nu_{X, J}^{\circ}$ et $\mu_{X}^{\circ}$ ainsi que d'une récurrence sur $\left|\boldsymbol{g}_{2}\right|$.

Par ailleurs il découle facilement de la proposition 3.1 que pour tout $\boldsymbol{f} \in \mathbf{N}^{I}$ tel que $\left\{i \in I, \quad f_{i} \geqslant 1\right\}=K$, on a $\nu_{X, J}^{\circ}(\boldsymbol{g}, \boldsymbol{f})=\nu_{X, J}^{\circ}\left(\boldsymbol{g}, \boldsymbol{f}_{K}\right)$.

Pour $\mathcal{D} \in \operatorname{Div}_{\text {eff }}(\mathscr{C})^{\mathfrak{I}}$, on pose

$$
\mu_{X}(\mathcal{D}) \stackrel{\text { déf }}{=} \prod_{v \in \mathscr{C}(0)} \mu_{X}^{\circ}(v(\mathcal{D})) .
$$

Pour $\mathcal{G} \in \operatorname{Div}_{\text {eff }}(\mathscr{C})^{J}$ et $\mathcal{D} \in \operatorname{Div}_{\text {eff }}(\mathscr{C})^{I}$, on pose

$$
\nu_{X, J}(\mathcal{G}, \mathcal{D}) \stackrel{\text { déf }}{=} \sum_{0 \leqslant \mathcal{D}^{\prime} \leqslant \mathcal{D}} \mu_{X}\left(\mathcal{G}, \mathcal{D}^{\prime}\right)=\prod_{v} \nu_{X, J}^{\circ}(v(\mathcal{G}, \mathcal{D})) .
$$

Remarque 3.4. - D'après la proposition $3.1, \nu_{X, J}(\mathcal{G}, \mathcal{D})$ est nul dès que $\mathcal{G} \notin$ $\operatorname{Div}_{\mathrm{eff}}(\mathscr{C})_{\leqslant 1}^{J}$.

Si $k=\mathbf{Q}$, on désignera par $\mu_{X, p, r}$ et $\nu_{X, J, p, r}$ les fonctions sur $\operatorname{Div}_{\text {eff }}\left(\mathscr{C}_{p, r}\right)^{\mathfrak{J}}$ obtenues en remplaçant dans les définitions ci-dessus $\mathscr{C}$ par $\mathscr{C}_{p, r}$. 
3.2. Hypersurfaces intrinsèques linéaires. - On conserve les hypothèses et notations introduites précédemment. On dit que $X$ est une hypersurface intrinsèque linéaire si $\mathscr{I}_{X}$ est principal et la propriété suivante est vérifiée : il existe $J \subset \mathfrak{I}$ et une famille $\left\{I_{j}\right\}_{j \in J}$ de parties non vide deux à deux disjointes de $\mathfrak{I} \backslash J$ tels que les classes $\left\{\mathcal{E}_{i}\right\}_{i \in \mathfrak{I} \backslash J}$ forment une base $\operatorname{de} \operatorname{Pic}(X)$ et, après une renormalisation éventuelle des $\left\{s_{i}\right\}_{i \in \mathfrak{I}}$, un générateur de $\mathscr{I}_{X}$ est de la forme

$$
\mathcal{F}=\sum_{j \in J} s_{j} \prod_{i \in I_{j}} s_{i}^{b_{i, j}}, \quad b_{i, j} \in \mathbf{N}_{>0}
$$

Tout $J \subset \mathfrak{I}$ vérifiant la propriété précédente est appelé un choix admissible de variables.

Exemple 3.5. - D'après [Der06], les 21 surfaces de del Pezzo généralisées de degré $\geqslant 3$ dont l'anneau total de coordonnées n'a qu'une équation sont toutes, à une exception près (l'une des deux cubiques avec singularité $\mathbf{D}_{4}$ apparaissant dans la classification), des hypersurfaces intrinsèques linéaires. Dans [Bou11a] est construite une famille d'hypersurfaces intrinsèques linéaires de dimension non bornée.

On notera $\mathscr{D}_{\text {tot }} \in \operatorname{Pic}(X)$ le degré d'un générateur de $\mathscr{I}_{X}$. D'après [BH07, proposition 8.5], on a la formule d'adjonction suivante.

Lemme 3.6. - La classe du fibré anticanonique de $X$ est

$$
-\mathscr{K}_{X}=\sum_{i \in \mathfrak{I}} \mathscr{E}_{i}-\mathscr{D}_{\text {tot }}
$$

3.3. Nombre de points sur le torseur universel. - Soit $X$ une hypersurface intrinsèque linéaire. On note $\mathscr{T}_{X}$ le schéma affine $\operatorname{Spec}\left(\mathbf{Z}\left[s_{i}\right]_{i \in \mathfrak{I}} / \mathcal{F}\right)$ où $\mathcal{F}$ est de la forme (3.2.1). Pour $e \in\{0,1\}^{\mathfrak{I}}$ on pose

$$
\mathscr{T}_{X, \boldsymbol{e}}=\mathscr{T}_{X} \cap \underset{i \in \mathfrak{I}, e_{i}=1}{\cap}\left\{s_{i}=0\right\} .
$$

Lemme 3.7. - Soit $k$ un corps fini de cardinal q. Soit $J$ un choix admissible de variables et $\boldsymbol{e}=(\boldsymbol{f}, \boldsymbol{g}) \in\{0,1\}^{\mathfrak{I}}$. On a

$$
\frac{\# \mathscr{T}_{X, \boldsymbol{e}}(k)}{q^{\operatorname{dim}\left(\mathscr{T}_{X}\right)}}=q^{-|\boldsymbol{e}|}\left[1+(q-1) \prod_{g_{j}+\sum_{i \in I_{j}} f_{i}=0}\left(1-\left(1-q^{-1}\right)^{\# I_{j}}\right)\right] .
$$

Démonstration. - On peut supposer que $\boldsymbol{e}=0$ et que $\cup_{j \in J} I_{j}=I$, les autres cas s'en déduisent facilement. Soit $\left(s_{i}\right) \in k^{I}$. Si pour tout $j \in J$ il existe $i \in I_{j}$ tel que $s_{i}=0$ le nombre de solutions $\left(s_{j}\right) \in k^{J}$ de l'équation linéaire $\mathcal{F}\left(s_{i}, s_{j}\right)=0$ est $q^{\# J}$. Dans le cas contraire, c'est $q^{\# J-1}$. En sommant sur tous les $\left(s_{i}\right) \in k^{I}$, on obtient la relation

$$
\# \mathscr{T}_{X}(k)=q^{\# J} \prod_{j \in J}\left(q^{\# I_{j}}-(q-1)^{\# I_{j}}\right)+q^{\# J-1}\left[q^{\# I}-\prod_{j \in J}\left(q^{\# I_{j}}-(q-1)^{\# I_{j}}\right)\right]
$$


Compte tenu de $\operatorname{dim} \mathscr{T}_{X}=\# \mathfrak{I}-1=\# I+\# J-1$, on en déduit bien la formule (3.3.2).

3.4. Relèvement du problème de comptage. - On conserve les hypothèses et notations précédentes. Désormais, le corps de bas $k$ sera toujours soit un corps fini de cardinal $q$ (avec en vue l'étude de «Manin I» et «Manin II »), soit le corps $\mathbf{Q}$ des rationnels (avec en vue l'étude de «Manin géométrique »; comme nous l'avons déjà signalé, la stratégie utilisée est en fait applicable à un corps de base quelconque mais nous nous limitons au cas des rationnels par souci de simplicité et parce que les exemples explicites d'application de notre résultat tombent dans ce cadre).

Soit $J \subset \mathfrak{I}$ un choix admissible de variables. On notera alors $I \stackrel{\text { déf }}{=} \mathfrak{I} \backslash J$. Écrivons, pour $j \in J$,

$$
\mathscr{G}_{j}=\sum_{i \in I} a_{i, j} \mathscr{F}_{i}, \quad a_{i, j} \in \mathbf{Z} .
$$

Pour $\mathcal{D} \in \operatorname{Div}_{\text {eff }}(\mathscr{C})$, on note $s_{\mathcal{D}}$ la section canonique de $\mathscr{O}_{\mathscr{C}}(\mathcal{D})$.

On suppose $k$ fini. Pour $\mathcal{D} \in \operatorname{Div}_{\text {eff }}(\mathscr{C})^{I}$ et $\mathcal{G} \in \operatorname{Div}_{\text {eff }}(\mathscr{C})^{J}$, on désigne par $\mathscr{N}(\mathcal{D}, \mathcal{G})$ (respectivement $\mathscr{N}^{*}(\mathcal{D}, \mathcal{G})$, respectivement $\mathscr{N}_{j_{0}}(\mathcal{D}, \mathcal{G})$ si $j_{0} \in J$ le cardinal de l'ensemble des éléments $\left(t_{j}\right)_{j \in J}$ (respectivement des éléments $\left(t_{j}\right)_{j \in J}$ tous non nuls, respectivement des éléments $\left(t_{j}\right)_{j \in J}$ avec $\left.t_{j_{0}}=0\right)$ du produit

$$
\prod_{j \in J} H^{0}\left(\mathscr{C}, \mathscr{O}_{\mathscr{C}}\left(-\mathcal{G}_{j}+\sum_{i \in I} a_{i, j} \mathcal{D}_{i}\right)\right)
$$

vérifiant la relation

$$
\mathcal{F}\left(s_{\mathcal{D}_{i}}, t_{j} s_{\mathcal{G}_{j}}\right)=0 .
$$

Si $k=\mathbf{Q}$, on définit de même les fonctions $\mathscr{N}_{p, r}, \mathscr{N}_{p, r}^{*}$ et $\mathscr{N}_{j_{0}, p, r}$ en remplaçant dans ce qui précède $\mathscr{C}$ par $\mathscr{C}_{p, r}$.

Supposons $k$ fini. Des résultats des sections 1.3, 1.4 et 1.6 de [Bou09a] découlent alors, pour tout $y \in \operatorname{Pic}(X)^{\vee} \cap C_{\text {eff }}(X)^{\vee}$, la formule

$$
\# \operatorname{Hom}_{X_{0}}(\mathscr{C}, X, y)(k)=\sum_{\begin{array}{c}
\mathcal{E} \in \operatorname{Div}_{\text {eff }}(\mathscr{C})^{\mathfrak{I}} \\
\operatorname{deg}\left(\mathcal{G}_{j}\right) \leqslant\left\langle y, \mathscr{G}_{j}\right\rangle, \quad j \in J \\
\operatorname{deg}\left(\mathcal{F}_{i}\right) \leqslant\left\langle y, \mathscr{F}_{i}\right\rangle, \quad i \in I \\
\operatorname{deg}\left(\mathcal{D}_{i}\right)=\left\langle y, \mathscr{F}_{i}\right\rangle-\operatorname{deg}\left(\mathcal{F}_{i}\right), \quad i \in I
\end{array}} \mu_{X}(\mathcal{G}, \mathcal{F}) \mathscr{N}^{*}(\mathcal{D}+\mathcal{F}, \mathcal{G}) .
$$

Pour alléger l'écriture, on adoptera dans toute la suite de cet article la notation suivante : soit $f$ une fonction à valeurs complexes (respectivement $\mathscr{P}$ une fonction booléenne) définie sur $\left(\operatorname{Pic}(X)^{\vee} \cap C_{\text {eff }}(X)^{\vee}\right) \times \operatorname{Div}_{\text {eff }}(\mathscr{C})^{\mathfrak{I}}$. On pose alors

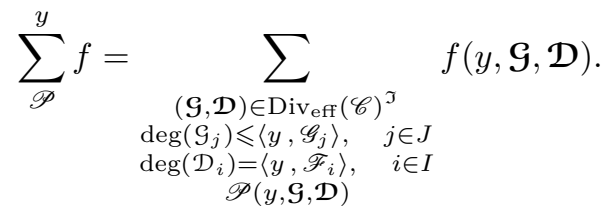


Ainsi d'après (3.4.4) et (3.1.7), on a

$$
\# \operatorname{Hom}_{X_{0}}(\mathscr{C}, X, y)(k)=\sum^{y} \nu_{X, J} \cdot \mathscr{N}^{*} .
$$

De même, si $k=\mathbf{Q}$, on adopte pour tous $p, r$ une notation analogue à (3.4.5) en remplaçant $\mathscr{C}$ par $\mathscr{C}_{p, r}$, et on a

$$
\# \operatorname{Hom}_{X_{0}}(\mathscr{C}, X, y)\left(\mathbf{F}_{p^{r}}\right)=\sum^{y} \nu_{X, J, p, r} \cdot \mathscr{N}_{p, r}^{*} .
$$

\section{4. Étude de quelques séries génératrices}

Proposition 4.1. - Soit $J$ un ensemble fini non vide.

1. Soit $I$ un ensemble fini non vide et $I=\bigsqcup_{j \in J} I_{j}$ une partition de $I$ en ensembles non vides. Soit $\boldsymbol{a} \in \mathbf{N}_{>0}^{I}, \boldsymbol{\nu} \in \mathbf{N}^{J}$ et

$$
\mathcal{F}(\rho, \boldsymbol{a}, \boldsymbol{\nu}, \boldsymbol{t}) \stackrel{\text { déf }}{=} \sum_{\boldsymbol{d} \in \mathbf{N}^{I}} \rho^{\operatorname{Min}\left(\nu_{j}+\sum_{i \in I_{j}} a_{i} d_{i}\right)} \boldsymbol{t}^{\boldsymbol{d}}
$$

(cette notation fait abstraction de la partition utilisée, qui sera toujours clairement indiquée par le contexte). Soit $\mathscr{I}$ la classe des parties $K$ de I telles que $K \cap I_{j}$ soit un singleton pour tout $j \in J$. Pour tout $K \in \mathscr{I}$ soit $m_{K}$ le plus petit commun multiple des $\left(a_{i}\right)_{i \in K}$. Soit

$$
\widetilde{\mathcal{F}}(\rho, \boldsymbol{a}, \boldsymbol{\nu}, \boldsymbol{t}) \stackrel{\text { déf }}{=} \prod_{K \in \mathscr{I}}\left(1-\rho^{m_{K}} \prod_{i \in K} t_{i}^{\frac{m_{K}}{a_{i}}}\right) \prod_{i \in I}\left(1-t_{i}\right) \mathcal{F}(\rho, \boldsymbol{a}, \boldsymbol{\nu}, \boldsymbol{t}) \quad \in \mathbf{Z}[\rho][[\boldsymbol{t}]] \text {. }
$$

(a) $\widetilde{\mathcal{F}}(\rho, \boldsymbol{a}, \boldsymbol{\nu}, \boldsymbol{t})$ est polynômial en $\rho$ et $\boldsymbol{t}$.

(b) On suppose qu'il existe $j_{0} \in J$ tel que $\nu_{j_{0}}=0$ et $a_{i}=1$ pour tout $i \in I_{j_{0}}$. On a alors

$$
\operatorname{deg}_{\rho, \boldsymbol{t}} \widetilde{\mathcal{F}}\left(\rho, \boldsymbol{a}, \boldsymbol{\nu}, \boldsymbol{t}^{-1}\right) \leqslant 0 .
$$

(c) Pour tout $\varepsilon>0$, il existe $\eta(\varepsilon)>0$ (dépendant de $\boldsymbol{a}$ et $\boldsymbol{\nu}$ ) tel qu'on ait pour tout $0 \leqslant \eta \leqslant \eta(\varepsilon)$

$$
\operatorname{deg}_{\rho, \boldsymbol{t}} \widetilde{\mathcal{F}}\left(\rho^{\eta}, \boldsymbol{a}, \boldsymbol{\nu}, \boldsymbol{t}^{-1}\right) \leqslant \varepsilon .
$$

2. Soit $j_{0} \in J, \boldsymbol{a} \in \mathbf{N}_{>0}^{J}, \boldsymbol{\nu} \in \mathbf{N}^{J}$ et

$$
\mathcal{G}\left(j_{0}, \rho, \tau, \boldsymbol{a}, \boldsymbol{\nu}, \boldsymbol{t}\right) \stackrel{\text { déf }}{=} \sum_{\boldsymbol{d} \in \mathbf{N}^{J}} \rho^{\operatorname{Min}_{j \in J}\left(a_{j} d_{j}+\nu_{j}\right)} \tau^{j \in J \backslash\left\{j_{0}\right\}} \boldsymbol{M i n}^{\left.\operatorname{Min}_{j} d_{j}+\nu_{j}\right)} \boldsymbol{t}^{\boldsymbol{d}} \in \mathbf{Z}[\rho, \tau][[\boldsymbol{t}]] \text {. }
$$

Soit $m$ le plus petit commun multiple des $\left(a_{j}\right)_{j \in J}$ et $n$ le plus petit commun multiple des $\left(a_{j}\right)_{j \in J \backslash\left\{j_{0}\right\}}$. Soit

$$
\widetilde{\mathcal{G}}\left(j_{0}, \rho, \tau, \boldsymbol{a}, \boldsymbol{\nu}, \boldsymbol{t}\right) \stackrel{\text { déf }}{=}\left(1-\rho^{m} \tau^{m} \prod_{j \in J} t_{j}^{\frac{m}{a_{j}}}\right)\left(1-\tau^{n} \prod_{j \in J \backslash\left\{j_{0}\right\}} t_{i}^{\frac{n}{a_{j}}}\right) \prod_{j \in J}\left(1-t_{j}\right) \mathcal{G}\left(j_{0}, \rho, \tau, \boldsymbol{a}, \boldsymbol{\nu}, \boldsymbol{t}\right) .
$$


(a) $\widetilde{\mathcal{G}}\left(j_{0}, \rho, \boldsymbol{a}, \boldsymbol{\nu}, \boldsymbol{t}\right)$ est polynômial en $\rho$ et $\boldsymbol{t}$.

(b) On suppose qu'il existe $j_{1} \in J$ tel que $\nu_{j_{1}}=0$ et $a_{j_{1}}=1$. Pour tout $\varepsilon>0$, il existe $\eta(\varepsilon)>0$ (dépendant de $\boldsymbol{a}$ et $\boldsymbol{\nu}$ ) tel qu'on ait pour tout $0 \leqslant \eta \leqslant \eta(\varepsilon)$

$$
\operatorname{deg}_{\rho, \boldsymbol{t}} \widetilde{\mathcal{G}}\left(j_{0}, \rho, \rho^{\eta}, \boldsymbol{a}, \boldsymbol{\nu}, \boldsymbol{t}^{-1}\right) \leqslant \varepsilon
$$

Démonstration. - Nous ne démontrons que la partie concernant $\mathcal{F}$. La partie concernant $\mathcal{G}$ se démontre en utilisant la même technique, laquelle est d'ailleurs une généralisation de celle utilisée dans la démonstration de [Bou11a, proposition 56].

Un calcul élémentaire montre que le coefficient $\alpha(\rho, \boldsymbol{a}, \boldsymbol{\nu}, \boldsymbol{d})$ de $\boldsymbol{t}^{\boldsymbol{d}}$ dans $\widetilde{\mathcal{F}}(\rho, \boldsymbol{a}, \boldsymbol{\nu}, \boldsymbol{t})$ vaut

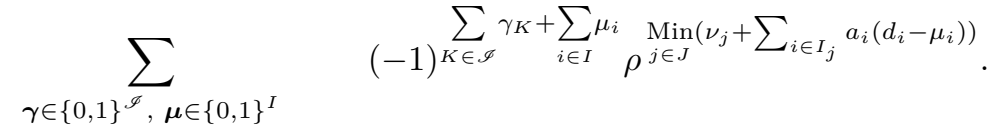

$$
\begin{aligned}
& \mu_{i}+\frac{1}{a_{i}} \sum_{\substack{K \in \mathscr{I} \\
i \in K}} m_{K} \cdot \gamma_{K} \leqslant d_{i}, \quad i \in I
\end{aligned}
$$

Soit $\boldsymbol{d} \in \mathbf{N}^{I}$. Pour tout $i \in I$, on note $\mathscr{P}(i)$ l'inégalité

$$
d_{i} \geqslant 1+\frac{1}{a_{i}} \sum_{\substack{K^{\prime} \in \mathscr{I} \\ i \in K^{\prime}}} m_{K}
$$

Supposons tout d'abord qu'il existe $K \in \mathscr{I}$ tel que tout $i \in K$ vérifie $\mathscr{P}(i)$. Alors les inégalités définissant le domaine de sommation dans (4.0.15) et faisant intervenir $\gamma_{K}$ sont toujours vérifiées; on en déduit aussitôt, en sommant par rapport à $\gamma_{K}$, que $\alpha(\rho, \boldsymbol{a}, \boldsymbol{\nu}, \boldsymbol{d})$ est nul.

On suppose à présent qu'il existe $j_{0} \in J$ et $i_{0} \in I_{j_{0}}$ tels qu'on ait

$$
d_{i_{0}} \geqslant 1+\frac{1}{a_{i_{0}}}\left(\sum_{K \in \mathscr{I}} m_{K}+\operatorname{Max}_{j \in J}\left|\nu_{j}-\nu_{j_{0}}\right|\right) .
$$

Montrons que $\alpha(\rho, \boldsymbol{a}, \boldsymbol{\nu}, \boldsymbol{d})$ est nul, ce qui établira le caractère polynomial de $\widetilde{\mathcal{F}}(\rho, \boldsymbol{a}, \boldsymbol{\nu}, \boldsymbol{t})$. D'après ce qui précède, on peut supposer que pour tout $K \in \mathscr{I}$ il existe $i \in K$ ne vérifiant pas $\mathscr{P}(i)$. On en déduit l'existence de $j_{1} \in J$ tel qu'aucun $i$ de $I_{j_{1}}$ ne vérifie $\mathscr{P}(i)$ (si un tel $j_{1}$ n'existait pas, on pourrait construire facilement un $K \in \mathscr{I}$ contredisant la propriété précédente). On a alors

$$
\sum_{i \in I_{j_{1}}} a_{i} d_{i} \leqslant \sum_{K \in \mathscr{I}} m_{K}
$$

et donc d'après (4.0.17)

$$
a_{i_{0}}\left(d_{i_{0}}-1\right)+\nu_{j_{0}} \geqslant \sum_{i \in I_{j_{1}}} a_{i} d_{i}+\nu_{j} .
$$


On en déduit aussitôt qu'on a pour tous les $(\boldsymbol{\gamma}, \boldsymbol{\mu}) \in\{0,1\}^{\mathscr{I}} \times\{0,1\}^{I}$ vérifiant les inégalités du domaine de sommation de (4.0.15) la relation

$$
\sum_{i \in I_{j_{0}}} a_{i}\left(d_{i}-\mu_{i}\right)+\nu_{j_{0}} \geqslant \sum_{i \in I_{j_{1}}} a_{i} d_{i}+\nu_{j_{1}} .
$$

Ainsi, dans (4.0.15), on peut remplacer $\operatorname{Min}_{j \in J} \operatorname{par} \underset{j \in J, j \neq j_{0}}{\operatorname{Min}} ;$ par ailleurs, d'après (4.0.17), la condition

$$
\mu_{i_{0}}+\frac{1}{a_{i_{0}}} \sum_{\substack{K \in \mathscr{G} \\ i_{0} \in K}} m_{K} \cdot \gamma_{K} \leqslant d_{i_{0}}
$$

est toujours vérifiée. On en déduit aussitôt, en sommant par rapport à $\mu_{i_{0}}$, que $\alpha(\rho, \boldsymbol{a}, \boldsymbol{\nu}, \boldsymbol{d})$ est nul.

$\mathrm{Vu}$ (4.0.15), le deuxième point découle de l'inégalité évidente

$$
\forall \boldsymbol{d} \in \mathbf{N}^{I}, \quad \nu_{j_{0}}+\sum_{i \in I_{j_{0}}} a_{i} d_{i}-|\boldsymbol{d}| \leqslant 0 .
$$

De même, pour le troisième point, il suffit de vérifier que si $\eta$ est assez petit on a pour tout $\boldsymbol{d} \in \mathbf{N}^{I}$

$$
\eta \operatorname{Min}_{j \in J}\left(\nu_{j}+\sum_{i \in I_{j}} a_{i} d_{i}\right)-|\boldsymbol{d}| \leqslant \varepsilon
$$

Définition 4.2. - Soit $M \in \mathbf{Z}$. Un élément $F$ de $\mathbf{Z}[\rho, \tau][[\boldsymbol{t}]]$ est dit $M$-contrôlé s'il s'écrit comme une somme finie d'éléments de la forme

$$
\frac{P(\rho, \tau, \boldsymbol{t})}{\prod_{\alpha \in A}\left(1-\rho^{m_{\alpha}} \tau^{n_{\alpha}} \prod_{i \in I} t_{i}^{d_{i_{\alpha}}}\right)}
$$

où $P \in \mathbf{Z}[\rho, \tau, \boldsymbol{t}], A$ est un ensemble fini (éventuellement vide) et pour $\alpha \in A, m_{\alpha}, n_{\alpha}$ et les $d_{i_{\alpha}}$ sont des entiers positifs, tels qu'on ait les propriétés suivantes : pour tout $\varepsilon>0$, il existe $\eta(\varepsilon)>0$ tel qu'on ait pour tout $0 \leqslant \eta(\varepsilon) \leqslant \eta$

$$
\operatorname{deg}_{\rho, \boldsymbol{t}} P\left(\rho, \rho^{\eta}, \boldsymbol{t}^{-1}\right) \leqslant M-2+\varepsilon ;
$$

et pour tout $\alpha \in A$

$$
m_{\alpha}-\sum_{i \in I} d_{i_{\alpha}} \leqslant-1
$$

Si $P \in \mathbf{N}[\rho, \tau, \boldsymbol{t}]$ la première condition est équivalente à $\operatorname{deg}_{\rho, t} P\left(\rho, 1, \boldsymbol{t}^{-1}\right) \leqslant M-2$.

Soit $H_{\bullet}=\left\{H_{\boldsymbol{g}}\right\}_{\boldsymbol{g} \in\{0,1\}^{I}}$ une famille d'éléments de $\mathbf{Z}[\rho, \tau][[\boldsymbol{t}]]$ telle que $H_{0}(\boldsymbol{t}=0)=$ 1. Soit $\eta \geqslant 0$. Si $k$ est fini, pour $\mathcal{G} \in \operatorname{Div}_{\mathrm{eff}}(\mathscr{C})_{\leqslant 1}^{J}$, on pose

$$
\sum_{\boldsymbol{d} \in \mathbf{N}^{I}} a_{\boldsymbol{d}, \eta}\left(H_{\bullet}, \mathcal{G}\right) \boldsymbol{t}^{d} \stackrel{\text { déf }}{=} \prod_{v \in \mathscr{C}(0)} \frac{H_{v(\mathcal{G})}\left(q_{v}, q_{v}^{\eta}, \boldsymbol{t}^{f_{v}}\right)}{\prod_{i \in I}\left(1-t_{i}^{f_{v}}\right)}
$$


et, si $k=\mathbf{Q}$, pour $\mathcal{G} \in \operatorname{Div}_{\mathrm{eff}}\left(\mathscr{C}_{p, r}\right)_{\leqslant 1}^{J}$, on pose

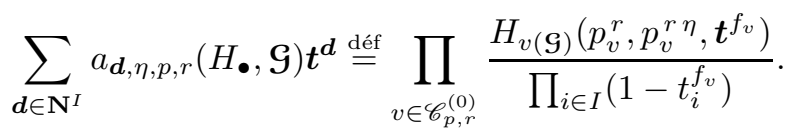

Lemme 4.3. - Avec les notations ci-dessus, on suppose que $H_{\boldsymbol{g}}$ est $|\boldsymbol{g}|$-contrôlé si $\boldsymbol{g} \in\{0,1\}^{J} \backslash\{0\}$ et que $H_{0}-1$ est 0 -contrôlé.

Il existe alors une constante $C>0$ telle que pour tout $\varepsilon>0$ assez petit, il existe $\eta(\varepsilon)>0$ (tout ceci pouvant être rendu explicite en fonction de $H_{\bullet}$ ) tels qu'on ait pour tout $\boldsymbol{d} \in \mathbf{N}^{I}$

1. ( $k$ fini) pour tout $\mathcal{G} \in \operatorname{Div}_{\mathrm{eff}}(\mathscr{C})_{\leqslant 1}^{J}$

(a)

$\left|a_{\boldsymbol{d}, 0}\left(H_{\bullet}, \mathcal{G}\right)-\left(\frac{h_{\mathscr{C}} q^{1-g_{\mathscr{C}}}}{q-1}\right)^{\# I} \prod_{v} H_{v(\mathcal{G})}\left(q_{v}, 1, q_{v}^{-1}\right) q^{|\boldsymbol{d}|}\right| \ll_{\varepsilon, H_{\bullet}}\left(\prod_{v, v(\mathcal{G}) \neq 0} C \cdot q_{v}^{|v(\mathcal{G})|-2+\varepsilon}\right)\left[\sum_{i \in I} q^{|\boldsymbol{d}|-\varepsilon d_{i}}\right]$

(b) pour tout $0 \leqslant \eta \leqslant \eta(\varepsilon)$

$$
a_{\boldsymbol{d}, \eta}\left(H_{\bullet}, \mathcal{G}\right) \ll_{\varepsilon, H}\left(\prod_{v, v(\mathcal{G}) \neq 0} q_{v}^{|v(\mathcal{G})|-2+\varepsilon}\right) q^{|\boldsymbol{d}|} .
$$

2. $(k=\mathbf{Q})$ Il existe $c(p, r)>0$ et $C(p, r, \varepsilon)>0$ vérifiant $\lim _{r \rightarrow+\infty} c(p, r)=1$ et $\lim _{r \rightarrow+\infty} C(p, r, \varepsilon)=1$ tels qu'on a

(a)

$$
\left|a_{\boldsymbol{d}, p, r, 0}\left(H_{\bullet}, 0\right)-c(p, r) p^{r|\boldsymbol{d}|}\right| \ll_{H_{\bullet}} C(p, r, \varepsilon)\left[\sum_{i \in I} p^{r|\boldsymbol{d}|-\varepsilon d_{i}}\right]
$$

(b) pour tout $\mathcal{G} \in \operatorname{Div}_{\mathrm{eff}}\left(\mathscr{C}_{p, r}\right)_{\leqslant 1}^{J}$ et tout $0 \leqslant \eta \leqslant \eta(\varepsilon)$

$$
\left|a_{\boldsymbol{d}, \eta, p, r}\left(H_{\bullet}, \mathcal{G}\right)\right| \ll_{H_{\bullet}} C(p, r, \varepsilon)\left(\prod_{v, v(\mathcal{G}) \neq 0} C \cdot p_{v}^{r(|v(\mathcal{G})|-2+\varepsilon)}\right) p^{r|\boldsymbol{d}|}
$$

Démonstration. - Elle est contenue dans [Bou11a, p.18] si $k$ est fini et dans [Bou11b, Section 6] si $k=\mathbf{Q}$. Indiquons seulement les grandes lignes : si $k$ est fini, on applique [Bou11b, Lemma 5.1] (qui est essentiellement un avatar des estimations de Cauchy) à la série

$$
\mathcal{H}(\mathcal{G}, \boldsymbol{t}) \stackrel{\text { déf }}{=} \prod\left(1-q t_{i}\right) \sum_{\boldsymbol{d} \in \mathbf{N}^{I}} a_{\boldsymbol{d}, \eta}(G, \mathcal{G}) \boldsymbol{t}^{\boldsymbol{d}}=\mathcal{H}(0, \boldsymbol{t}) \prod_{v, v(\mathcal{G}) \neq 0} \frac{H_{v(\mathcal{G})}\left(q_{v}, q_{v}^{\eta}, \boldsymbol{t}^{f_{v}}\right)}{H_{0}\left(q_{v}, q_{v}^{\eta}, \boldsymbol{t}^{f_{v}}\right)} .
$$


Les hypothèses faites sur $H$ • permettent de majorer de manière ad hoc la norme sup

$$
\left\|\prod_{v, v(\mathcal{G}) \neq 0} \frac{H_{v(\mathcal{G})}\left(q_{v}, q_{v}^{\eta}, \boldsymbol{t}^{f_{v}}\right)}{H_{0}\left(q_{v}, q_{v}^{\eta}, \boldsymbol{t}^{f_{v}}\right)}\right\|_{q^{-1+\varepsilon}} .
$$

Si $k=\mathbf{Q}$ le raisonnement est analogue. Il faut montrer en outre dans ce cas qu'on

$$
\lim _{r \rightarrow+\infty} \widetilde{\mathcal{H}}\left(0,\left(p^{-r}, \ldots, p^{-r}\right)\right)=1
$$

et, pour $\varepsilon$ assez petit,

$$
\limsup _{r \rightarrow+\infty}\|\widetilde{\mathcal{H}}(0, \boldsymbol{t})\|_{p^{r(-1+\varepsilon)}}=1
$$

où $\widetilde{\mathcal{H}}(0, \boldsymbol{t}) \stackrel{\text { déf }}{=} \mathcal{H}(0, \boldsymbol{t}) \prod\left(1-t_{i}\right)$ ce qui se fait en utilisant la décomposition en produit eulérien et en appliquant [Bou11b, Lemma 5.2].

Désormais, on considère une hypersurface intrinsèque linéaire $X$ et un ensemble admissible de variables $J$ (cf. la sous-section 3.2). Rappelons qu'on a alors $\operatorname{dim}(X)+$ $1=\# J$ et $\# I=\operatorname{rk}(\operatorname{Pic}(X))$. Dans tout ce qui suit, si $A, B$ et $x$ sont des quantités, la notation $A \ll_{x} B$ signifiera « $A$ est majoré par $B$ à une constante multiplicative près ne dépendant que de $X, \mathscr{C}$ et $x »$. Les constantes introduites ne dépendront que de $X$ et $\mathscr{C}$.

Nous aurons besoin de l'hypothèse suivante sur $X$.

Hypothèse 4.4. - 1. Au plus $\operatorname{dim}(X)+1$ diviseurs parmi les $\left\{\mathcal{D}_{i}\right\}_{i \in \mathfrak{C}_{i n c}}$ s'intersectent mutuellement. En d'autres termes, on a $\operatorname{Max}\left\{\# K, K \in \mathcal{C}_{\text {inc }}\right\} \leqslant$ $\operatorname{dim} X+1$.

2. si $K \in \mathcal{C}_{\text {inc }}$ est tel que $K \cap I_{j}$ est un singleton pour tout $i$, alors il existe $i \in K$ tel que $a_{i}=1$.

Exemple 4.5. - On vérifie aussitôt que cette hypothèse est satisfaite pour les surfaces de la liste de [Der06], ainsi que pour la famille construite dans [Bou11a]. Nous ne savons pas s'il existe des exemples d'hypersurfaces linéaires intrinsèques pour lesquelles elle n'est pas vérifiée.

Supposons $k$ fini. Pour $\mathcal{G} \in \operatorname{Div}_{\text {eff }}(\mathscr{C})^{J}$ et $\boldsymbol{d} \in \mathbf{N}^{I}$, on pose

$$
\mathscr{M}(\boldsymbol{d}, \mathcal{G}) \stackrel{\text { déf }}{=} \sum_{\substack{\mathcal{D} \in \operatorname{Div}_{\text {eff }}(\mathscr{C})^{I} \\ \operatorname{deg}(\mathcal{D})=d}} \nu_{X, J}(\mathcal{G}, \mathcal{D}) q^{\operatorname{deg}\left(\operatorname{pgcd}_{j \in J}\left(s_{\mathcal{G}_{j}} \prod_{i \in I_{j}} s_{\mathcal{D}_{i}}^{a_{i}}\right)\right)}
$$

et, pour $\eta \geqslant 0$ et $j_{0} \in J$,

$$
\mathscr{M}_{j_{0}, \eta}(\boldsymbol{d}, \mathcal{G}) \stackrel{\text { déf }}{=} \sum_{\substack{\mathcal{D} \in \operatorname{Div} \operatorname{vefl}_{(}(\mathscr{C})^{I} \\ \operatorname{deg}(\mathcal{D})=\boldsymbol{d}}}\left|\nu_{X, J}(\mathcal{G}, \mathcal{D})\right| q^{\operatorname{deg}\left(\operatorname{pgcd}_{j \in J}\left(s_{\mathcal{G}_{j}} \prod_{i \in I_{j}} s_{\mathcal{D}_{i}}^{a_{i}}\right)\right)+\eta \operatorname{deg}\left(\operatorname{pgcd}_{j \in J \backslash\left\{j_{0}\right\}}\left(s_{\mathcal{G}_{j}} \prod_{i \in I_{j}} s_{\mathcal{D}_{i}}^{a_{i}}\right)\right)} .
$$

Notons que d'après la remarque 3.4 , ces quantités sont nulles si $\mathcal{G} \notin \operatorname{Div}_{\text {eff }}(\mathscr{C})_{\leqslant 1}^{J}$. 
Si $k=\mathbf{Q}$, on définit de même les fonctions $\mathscr{M}_{p, r}$ et $\mathscr{M}_{j_{0}, \eta, p, r}$ sur $\mathbf{N}^{I} \times \operatorname{Div}_{\text {eff }}\left(\mathscr{C}_{p, r}\right)^{J}$ en remplaçant dans les formules ci-dessus $\mathscr{C}$ par $\mathscr{C}_{p, r}, \nu_{X, J}$ par $\nu_{X, J, p, r}$ et $q$ par $p^{r}$.

Posons $\mathscr{F}(n)=\operatorname{Min}(1, n)$. Pour $\boldsymbol{g} \in \mathbf{N}^{J}$, soit

$$
\begin{gathered}
F_{\boldsymbol{g}}(\rho, \boldsymbol{t}) \stackrel{\text { déf }}{=} \sum_{\boldsymbol{f} \in \mathbf{N}^{I}} \nu_{X, J}^{\circ}(\boldsymbol{g}, \boldsymbol{f}) \rho^{\stackrel{\operatorname{Min}\left(g_{j}+\sum_{i \in I_{j}} a_{i} f_{i}\right)}{{ }^{\prime}}} \boldsymbol{t}^{\boldsymbol{f}}, \\
F_{1, \boldsymbol{g}}(\rho, \boldsymbol{t}) \stackrel{\text { déf }}{=} \sum_{\boldsymbol{f} \in \mathbf{N}^{I}} \nu_{X, J}^{\circ}(\boldsymbol{g}, \boldsymbol{f}) \rho \\
F_{2, \boldsymbol{g}}(\rho, \boldsymbol{t}) \stackrel{\text { déf }}{=} F_{\boldsymbol{g}}(\rho, \boldsymbol{t})-F_{1, \boldsymbol{g}}(\rho, \boldsymbol{t})
\end{gathered}
$$

et, pour $\alpha \in\{1,2\}$,

$$
H_{\alpha, \boldsymbol{g}}(\rho, \boldsymbol{t}) \stackrel{\text { déf }}{=}\left(\prod_{i \in I} 1-t_{i}\right) F_{\alpha, \boldsymbol{g}}(\rho, \boldsymbol{t}) \text {. }
$$

Pour $j_{0} \in J$ on pose

$$
F_{j_{0}, \boldsymbol{g}}(\rho, \tau, \boldsymbol{t}) \stackrel{\text { déf }}{=} \sum_{\boldsymbol{f} \in \mathbf{N}^{I}}\left|\nu_{X, J}^{\circ}(\boldsymbol{g}, \boldsymbol{f})\right| \rho^{\left.\stackrel{\operatorname{Min}\left(g_{j}+\sum_{i \in I_{j}} a_{i} f_{i}\right)}{{ }^{j \in J}} \tau^{j \in J \backslash\left\{j_{0}\right\}} \operatorname{Min}_{j}+\sum_{i \in I_{j}} a_{i} f_{i}\right)} \boldsymbol{t}^{\boldsymbol{f}},
$$

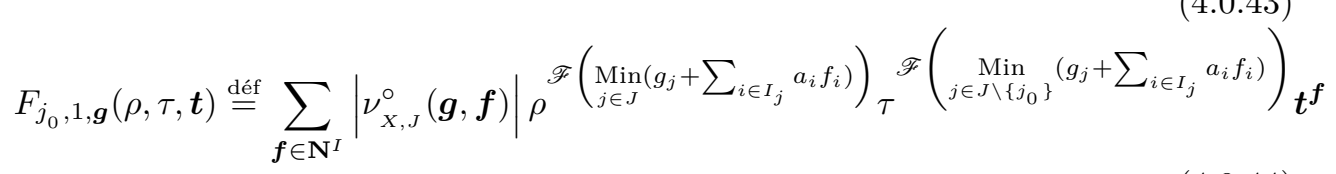

et

$$
F_{j_{0}, 2, \boldsymbol{g}} \stackrel{\text { déf }}{=} F_{j_{0}, \boldsymbol{g}}-F_{j_{0}, 1, \boldsymbol{g}}
$$

Proposition 4.6. - On suppose l'hypothèse 4.4 vérifiée.

1. Pour tout $\boldsymbol{g} \in\{0,1\}^{J}, H_{1, \boldsymbol{g}}$ est un élément de $\mathbf{Z}[\rho, \boldsymbol{t}] . H_{1,0}-1$ est 0 -contrôlée et $H_{1, \boldsymbol{g}}$ est $|\boldsymbol{g}|$-contrôlée pour $\boldsymbol{g} \neq 0$

2. Pour tout $\boldsymbol{g} \in\{0,1\}^{J}, H_{2, \boldsymbol{g}}$ est $|\boldsymbol{g}|$-contrôlée.

3. Il existe un élément $H_{j_{0}, 1, \boldsymbol{g}} \in \mathbf{N}[\rho, \tau, \boldsymbol{t}]$ tel que $H_{j_{0}, 1,0}-1$ est 0 -contrôlé, $H_{j_{0}, 1, \boldsymbol{g}}$ est $|\boldsymbol{g}|$-contrôlée pour $\boldsymbol{g} \neq 0$, et pour tout $\rho, \tau>0$

$$
F_{j_{0}, 1, \boldsymbol{g}}(\rho, \tau, \boldsymbol{t})\left(\prod_{i \in I} 1-t_{i}\right) \leqslant H_{j_{0}, 1, \boldsymbol{g}}(\rho, \tau, \boldsymbol{t}) .
$$

4. Pour tout $\boldsymbol{g} \in\{0,1\}^{J}, F_{j_{0}, 2, \boldsymbol{g}}$ est $|\boldsymbol{g}|$-contrôlé.

5. (k fini) Pour tout $\mathcal{G} \in \operatorname{Div}_{\mathrm{eff}}(\mathscr{C})_{\leqslant 1}^{J}$, posons

$$
c_{\text {princ }}(\mathcal{G}) \stackrel{\text { déf }}{=}\left(\frac{h_{\mathscr{C}} q^{1-g_{\mathscr{C}}}}{q-1}\right)^{\# I} \prod_{v \in \mathscr{C}(0)} H_{v(\mathcal{G})}\left(q_{v}, q_{v}^{-1}\right)
$$


Il existe alors $C>0$ telle que pour tout $\varepsilon>0$ assez petit, il existe $\eta(\varepsilon)>0$ (tout ceci pouvant être explicitement décrit en fonction de $X$ ) tels qu'on ait pour tout $\boldsymbol{d} \in \mathbf{N}^{I}$, et tout $\boldsymbol{G} \in \operatorname{Div}_{\mathrm{eff}}(\mathscr{C})_{\leqslant 1}^{J}$

$\left|q^{-|\boldsymbol{d}|} \mathscr{M}(\boldsymbol{d}, \mathcal{G})-c_{\text {princ }}(\mathcal{G})\right| \ll_{\varepsilon}\left(\prod_{v, v(\mathcal{G}) \neq 0} C \cdot q_{v}^{|v(\mathcal{G})|-2+\varepsilon}\right)\left[\sum_{i \in I} q^{-\varepsilon d_{i}}\right]$

et pour tout $0 \leqslant \eta \leqslant \eta(\varepsilon)$

$$
q^{-|\boldsymbol{d}|}\left|\mathscr{M}_{j_{0}, \eta}(\boldsymbol{d}, \mathcal{G})\right| \ll_{\varepsilon}\left(\prod_{v, v(\mathcal{G}) \neq 0} C \cdot q_{v}^{|v(\mathcal{G})|-2+\varepsilon}\right)
$$

Par ailleurs, on a

$$
q^{\left(1-g_{\mathscr{C}}\right) \operatorname{dim}(X)} \sum_{\mathcal{G} \in \operatorname{Div}_{\text {eff }}(\mathscr{C})_{\leqslant 1}^{J}} c_{\text {princ }}(\mathcal{G}) q^{-|\operatorname{deg}(\mathcal{G})|}=\gamma(X)
$$

et pour tout $\varepsilon>0$ assez petit

$$
\left|\gamma(X)-q^{\left(1-g_{\mathscr{C}}\right) \operatorname{dim}(X)} \sum_{\substack{\mathcal{G} \in \operatorname{Diveff}_{\text {ef }}(\mathscr{C})_{\leqslant 1}^{J} \\ \operatorname{deg}\left(\mathcal{G}_{j}\right) \leqslant\left\langle y, \mathscr{G}_{j}\right\rangle, j_{j \in J}}} c_{\text {princ }}(\mathcal{G}) q^{-|\operatorname{deg}(\mathcal{G})|}\right| \ll_{\varepsilon} q^{-\varepsilon \sum_{j \in J}\left\langle y, \mathscr{G}_{j}\right\rangle} .
$$

6. $(k=\mathbf{Q})$

Il existe $C>0$ et $c(p, r)>0$ telles que pour tout $\varepsilon>0$ assez petit il existe $C(p, r, \varepsilon)>0$ et $\eta(\varepsilon)>0$ (tout ceci pouvant être rendu explicite en fonction de $X)$, avec $\lim _{r \rightarrow+\infty} c(p, r)=1$ et $\lim _{r \rightarrow+\infty} C(p, r, \varepsilon)=1$ tels qu'on ait pour tout $\boldsymbol{d} \in \mathbf{N}^{I}$, pour tout $\mathcal{G} \in \operatorname{Div}_{\mathrm{eff}}\left(\mathscr{C}_{p, r}\right)_{\leqslant 1}^{J}$ et tout $0 \leqslant \eta \leqslant \eta(\varepsilon)$

$$
\begin{aligned}
& \left|p^{-r|\boldsymbol{d}|} \mathscr{M}_{p, r}(\boldsymbol{d}, 0)-c(p, r) p^{r|\boldsymbol{d}|}\right| \leqslant C(p, r, \varepsilon)\left[\sum_{i \in I} p^{\left.-\varepsilon d_{i}\right)}\right] \\
\text { et } \quad p^{-r|\boldsymbol{d}|} \mathscr{M}_{j_{0}, \eta, p, r}(\boldsymbol{d}, \mathcal{G}) \leqslant C(p, r, \varepsilon) & \left(\prod_{v, v(\mathcal{G}) \neq 0} C \cdot p_{v}^{r(|v(\mathcal{G})|-2+\varepsilon)}\right)
\end{aligned}
$$

Démonstration. — Compte tenu de (3.1.4), on a

$$
F_{1, \boldsymbol{g}}(\rho, \boldsymbol{t})=\sum_{\substack{\boldsymbol{m} \in\{0,1\}^{I} \\ \boldsymbol{f} \in \mathbf{N}^{I}}} \mu_{X}^{\circ}(\boldsymbol{g}, \boldsymbol{m}) \boldsymbol{t}^{\boldsymbol{m}+\boldsymbol{f}}+\sum_{\substack{\boldsymbol{m} \in\{0,1\}^{I}, \boldsymbol{f} \in \mathbf{N}^{I} \\ \forall j \in J,}} \mu_{X j}^{\circ}(\boldsymbol{g}, \boldsymbol{m})(\rho-1) \boldsymbol{t}^{\boldsymbol{m}+\boldsymbol{f}} .
$$


Or pour $\boldsymbol{m} \in\{0,1\}^{I}$ on a

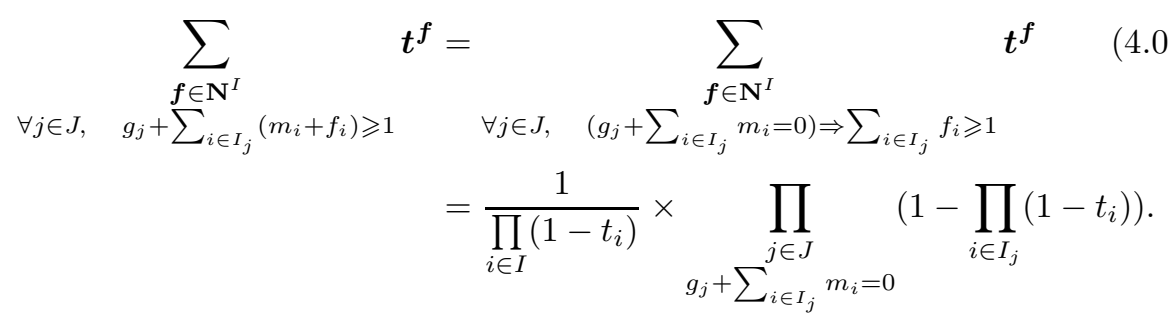

On en déduit l'égalité

$$
H_{1, \boldsymbol{g}}(\rho, \boldsymbol{t})=\sum_{\boldsymbol{m} \in\{0,1\}^{I}} \mu_{X}^{\circ}(\boldsymbol{g}, \boldsymbol{m}) \boldsymbol{t}^{\boldsymbol{m}}\left[1+(\rho-1) \prod_{j \in J}\left(1-\prod_{i \in I_{j}}\left(1-t_{i}\right)\right)\right]
$$

Mais pour tous $\boldsymbol{m}, \boldsymbol{g}$ on a

$$
\sum_{i \in I} m_{i}+\#\left\{j \in J, g_{j}+\sum_{i \in I_{j}} m_{j}=0\right\} \geqslant \#\left\{j \in J, g_{j}=0\right\}=\# J-|\boldsymbol{g}| \geqslant 3-|\boldsymbol{g}|
$$

On déduit de l'inégalité précédente et de (4.0.57) les majorations

$$
\operatorname{deg}_{\rho, \boldsymbol{t}} H_{1, \boldsymbol{g}}\left(\rho, \boldsymbol{t}^{-1}\right) \leqslant \operatorname{Max}\left(|\boldsymbol{g}|-2, \underset{\substack{\boldsymbol{m} \in\{0,1\}^{I} \\ \mu_{X}^{\circ}(\boldsymbol{m}, \boldsymbol{g}) \neq 0}}{\operatorname{Max}}(-|\boldsymbol{m}|)\right)
$$

et

$$
\operatorname{deg}_{\rho, \boldsymbol{t}}\left[H_{1,0}\left(\rho, \boldsymbol{t}^{-1}\right)-1\right] \leqslant \operatorname{Max}\left(-2, \operatorname{Max}_{\substack{\boldsymbol{m} \in\{0,1\}^{I} \\|\boldsymbol{m}| \neq 0 \\ \mu_{X}^{\circ}(\boldsymbol{m}, 0) \neq 0}}(-|\boldsymbol{m}|)\right)
$$

Soit $\boldsymbol{m} \in\{0,1\}^{I}$ et $\boldsymbol{g} \in\{0,1\}^{J}$ tels que $\mu_{X}^{\circ}(\boldsymbol{g}, \boldsymbol{m}) \neq 0$ et tels qu'en outre si $|\boldsymbol{g}|=0$ on a $|\boldsymbol{m}| \neq 0$. On a alors

$$
-|\boldsymbol{m}| \leqslant|\boldsymbol{g}|-2
$$

C'est en effet immédiat si $|\boldsymbol{g}| \geqslant 2$ et si $|\boldsymbol{g}|=1$ ou $|\boldsymbol{g}|=0$ cela découle de la proposition 3.1. Ceci conclut la démonstration du premier point.

Passons au deuxième point. La remarque 3.2 permet d'écrire

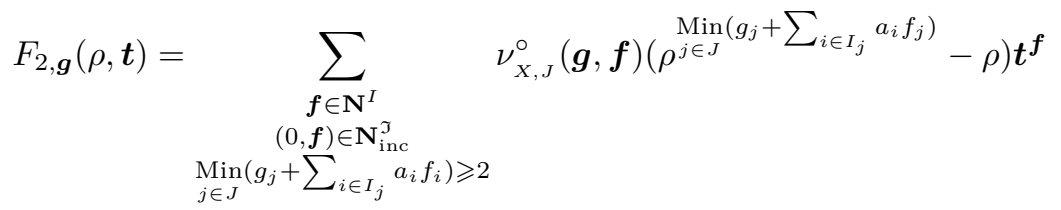

Soit $\mathscr{I}$ la classe des sous-ensembles $K \subset I$ de cardinal $\# J$ tel que $K \in \mathcal{C}_{\text {inc }}$ et $K \cap I_{j}$ est un singleton pour tout $j \in J$. Compte tenu du point 1 de l'hypothèse 4.4 , on voit alors que si $\boldsymbol{f} \in \mathbf{N}^{I}$ vérifie les conditions sous le signe somme dans (4.0.62), il existe 
$K \in \mathscr{I}$ tel que $f_{i} \geqslant 1$ si et seulement si $i \in K$. On a alors ( $c f$. la remarque 3.3 ) $\nu_{X, J}^{\circ}(\boldsymbol{g}, \boldsymbol{f})=\nu_{X, J}^{\circ}\left(\boldsymbol{g}, \boldsymbol{f}_{K}\right)$. La fonction $F_{2, \boldsymbol{g}}$ se réécrit donc comme une somme sur tous les $K \in \mathscr{I}$ des fonctions

$$
\nu_{X, J}^{\circ}\left(\boldsymbol{g}, \boldsymbol{f}_{K}\right) \sum_{\substack{\boldsymbol{f} \in \mathbf{N}_{>0}^{J} \\ j \in J}} \underbrace{\operatorname{Min}\left(g_{j}+a_{j} f_{j}\right)}_{\left(\rho_{j}+a_{j} f_{j}\right) \geqslant 2}-\rho) \boldsymbol{t}^{\boldsymbol{f}} .
$$

Pour alléger l'écriture, on a fixé dans l'expression précédente une identification de $K$ à $J$. Soit $J_{1} \stackrel{\text { déf }}{=}\left\{j \in J, a_{j}=1\right\}$. D'après le point 2 de l'hypothèse $4.4, J_{1}$ est non vide. Notons $m$ le plus petit commun multiple de la famille $\left(a_{j}\right)_{j \in J}$. L'expression (4.0.63) se réécrit alors, compte tenu de la proposition 4.1,

$$
\begin{aligned}
& \nu_{X, J}^{\circ}\left(\boldsymbol{g}, \boldsymbol{f}_{K}\right) \sum_{\substack{\boldsymbol{f} \in \mathbf{N}_{>0}^{K} \\
\forall j \in J_{1}, g_{j}=0 \Rightarrow f_{j} \geqslant 2}}\left(\rho^{\operatorname{Min}\left(g_{j}+a_{j} f_{j}\right)}-\rho\right) \boldsymbol{t}^{\boldsymbol{f}} \\
& =\nu_{X, J}^{\circ}\left(\boldsymbol{g}, \boldsymbol{f}_{K}\right) \prod_{j \in J_{1}} t_{j}^{2-g_{j}} \prod_{j \in J \backslash J_{1}} t_{j} \sum_{\boldsymbol{f} \in \mathbf{N}_{\geqslant 0}^{J}}\left(\rho^{2+\operatorname{Min}\left(\left(f_{j}\right)_{j \in J_{1}},\left(g_{j}+a_{j}-2+f_{j}\right)_{j \in J} \backslash J_{1}\right)}-\rho\right) \boldsymbol{t}^{\boldsymbol{f}} \\
& =\nu_{X, J}^{\circ}\left(\boldsymbol{g}, \boldsymbol{f}_{K}\right) \rho \prod_{j \in J_{1}} t_{j}^{2-g_{j}} \prod_{j \in J \backslash J_{1}} t_{j}\left[\frac{\rho \tilde{\mathcal{F}}\left(\rho,\left(a_{j}\right)_{j \in J}, \boldsymbol{\nu}, \boldsymbol{t}\right)}{\left(1-\rho^{m} \prod_{j \in J} t_{j}^{\frac{m}{a_{j}}}\right) \prod_{i \in J}\left(1-t_{j}\right)}-\frac{1}{\prod_{i \in J} 1-t_{j}}\right]
\end{aligned}
$$

avec $\boldsymbol{\nu}=\left((0, \ldots, 0)_{j \in J_{1}},\left(g_{j}+a_{j}-2\right)_{j \in J \backslash J_{1}}\right)$. Remarquons que si l'on fixe tous les paramètres sauf $\left(g_{j}\right) \in\{0,1\}^{J_{1}}$, l'expression précédente vaut à une constante près $\nu_{X, J}^{\circ}\left(\left(g_{j}\right)_{j \in J_{1}},\left(g_{j}\right)_{j \in J \backslash J_{1}}, \boldsymbol{f}_{K}\right) \prod_{j \in J_{1}} t_{j}^{-g_{j}}$.

D'après la proposition 4.1 , on a

$$
\operatorname{deg}_{\rho, t}\left(\widetilde{\mathcal{F}}\left(\rho,\left(a_{j}\right)_{j \in J}, \boldsymbol{\nu}, \boldsymbol{t}^{-1}\right)\right) \leqslant 0 .
$$

On a également

$$
\operatorname{deg}_{\rho, \boldsymbol{t}}\left(\rho \prod_{j \in J_{1}} t_{j}^{g_{j}-2} \prod_{j \in J \backslash J_{1}} t_{j}^{-1}\right)=1-\# J_{1}-\# J+\sum_{j \in J_{1}} g_{j} \leqslant|\boldsymbol{g}|-3
$$

(rappelons qu'on a $\# J_{1} \geqslant 1$ et $\# J \geqslant 3$ ).

Finalement, compte tenu du fait que $J_{1}$ est non vide, on a

$$
m-\sum_{i \in J} \frac{m}{a_{j}} \leqslant-\left(\# J_{1}-1\right)-\left(\# J-\# J_{1}\right) \leqslant-2 .
$$

Ceci conclut la démonstration du deuxième point. 
Pour le troisième point, on a la majoration

$$
F_{j_{0}, 1, \boldsymbol{g}}(\rho, \boldsymbol{t}) \leqslant \sum_{\boldsymbol{f} \in \mathbf{N}^{I}} \sum_{0 \leqslant \boldsymbol{f}^{\prime} \leqslant \boldsymbol{f}}\left|\mu_{X}^{\circ}\left(\boldsymbol{g}, \boldsymbol{f}^{\prime}\right)\right| \rho^{\mathscr{F}\left(\operatorname{Min}_{j \in J}\left(g_{j}+\sum_{i \in I_{j}} a_{i} f_{i}\right)\right)} \tau \tau_{j \in J \backslash\left\{j_{0}\right\}}^{\left.\mathscr{F}\left(g_{j}+\sum_{i \in I_{j}} a_{i} f_{i}\right)\right)} \boldsymbol{t}^{\boldsymbol{f}} .
$$

Notons $\frac{H_{j_{0}, 1, \boldsymbol{g}}(\rho, \boldsymbol{t})}{\prod_{i \in I}\left(1-t_{i}\right)}$ cette dernière expression. Un calcul similaire à celui mené pour le premier point montre qu'on a

$$
H_{j_{0}, 1, \boldsymbol{g}}(\rho, \boldsymbol{t})=\sum_{\boldsymbol{m} \in\{0,1\}^{I}}\left|\mu_{X}^{\circ}(\boldsymbol{g}, \boldsymbol{m})\right| \boldsymbol{t}^{\boldsymbol{m}} H_{\boldsymbol{m}, j_{0}, 1, \boldsymbol{g}}(\rho, \tau, \boldsymbol{t})
$$

avec, pour tous $\boldsymbol{g}, \boldsymbol{m}$,

$$
H_{\boldsymbol{m}, j_{0}, 1, \boldsymbol{g}}(\rho, \tau, \boldsymbol{t})=1+(\rho \tau-1) \prod_{g_{j}+\sum_{i \in I_{j}} m_{i}=0}\left(1-\prod_{i \in I_{j}}\left(1-t_{i}\right)\right)
$$

si $g_{j_{0}} \neq 0$ ou s'il existe $i \in I_{j_{0}}$ tel que $m_{i} \neq 0$, et

$$
H_{\boldsymbol{m}, j_{0}, 1, \boldsymbol{g}}(\rho, \tau, \boldsymbol{t})=1+(\rho \tau-1) \prod_{\substack{j \in J \\ g_{j}+\sum_{i \in I_{j}} m_{i}=0}}\left(1-\prod_{i \in I_{j}}\left(1-t_{i}\right)\right)+\tau \prod_{i \in I_{j_{0}}}\left(1-t_{i}\right) \prod_{\substack{j \in J \backslash\left\{j_{0}\right\} \\ g_{j}+\sum_{i \in I_{j}} m_{i}=0}}\left(1-\prod_{i \in I_{j}}\left(1-t_{i}\right)\right)
$$

si $g_{j_{0}}=0$ et pour tout $i \in I_{j_{0}}, m_{i}=0$. On conclut de manière similaire au premier point.

La démonstration du quatrième point est reportée dans un appendice. L'idée est similaire à celle utilisée pour le deuxième point, avec quelques complications techniques.

Les deux derniers points se déduisent aussitôt des quatre premiers par application du lemme 4.3, à l'exception de (4.0.50) et (4.0.51). Or on a

$$
\sum_{\mathcal{G} \in \operatorname{Diveff}_{(\mathscr{C})^{J}}} \prod_{v} H_{v(\mathcal{G})}\left(q_{v}, q_{v}^{-1}\right) q^{-|\operatorname{deg}(\mathcal{G})|}=\prod_{v} \sum_{\boldsymbol{g} \in\{0,1\}^{J}} H_{\boldsymbol{g}}\left(q_{v}, q_{v}^{-1}\right) q^{-|\boldsymbol{g}|} .
$$

Tout d'abord, d'après la remarque qui suit (4.0.66) et la remarque 3.3, on a

$$
\sum_{\boldsymbol{g} \in\{0,1\}^{J}} H_{2, \boldsymbol{g}}\left(q_{v}, q_{v}^{-1}\right) q^{-|\boldsymbol{g}|}=0
$$

Par ailleurs, d'après (4.0.57), on a

$$
\sum_{\boldsymbol{g} \in\{0,1\}^{J}} H_{1, \boldsymbol{g}}\left(q_{v}, q_{v}^{-1}\right) q^{-|\boldsymbol{g}|}=\sum_{\boldsymbol{e}=(\boldsymbol{g}, \boldsymbol{f}) \in\{0,1\}\}^{I \cup J}} \mu_{X}^{\circ}(\boldsymbol{e}) q_{v}^{-|\boldsymbol{e}|}\left[1+\left(q_{v}-1\right) \prod_{j \in J}\left(1-\left(1-q_{v}^{-1}\right)^{\# I_{j}}\right)\right]
$$


D'après le lemme 3.7 et [Bou09a, Lemme 1.25], on a donc

$\sum_{\boldsymbol{g}\{0,1\}^{J}} H_{\boldsymbol{g}}\left(q_{v}, q_{v}^{-1}\right) q^{-|\boldsymbol{g}|}=\sum_{\boldsymbol{e}=(\boldsymbol{g}, \boldsymbol{f}) \in\{0,1\}^{I \cup J}} \mu_{X}^{\circ}(\boldsymbol{e}) \frac{\# \mathscr{T}_{X, \boldsymbol{e}}\left(\kappa_{v}\right)}{q_{v}^{\operatorname{dim}\left(\mathscr{T}_{X}\right)}}=\left(1-q_{v}^{-1}\right)^{\operatorname{rg}(\operatorname{Pic}(X))} \frac{\# X\left(\kappa_{v}\right)}{q_{v}^{\operatorname{dim}(X)}}$

d'où $(4.0 .50) ;(4.0 .51)$ en découle facilement.

\section{Comptage de sections globales}

On considère une hypersurface intrinsèque linéaire $X$ et un ensemble admissible de variables $J$ (cf. la sous-section 3.2). On suppose en outre que $\operatorname{dim}(X)=2$ (en d'autres termes que $\# J=3)$. Pour $j_{0} \in J, y \in \operatorname{Pic}(X)^{\vee}$ et $(\mathcal{G}, \mathcal{D}) \in \operatorname{Div}_{\text {eff }}(\mathscr{C})^{J \times I}$, on pose

$$
\begin{gathered}
\varphi_{j_{0}}(y, \mathcal{G}, \mathcal{D}) \stackrel{\text { déf }}{=}\left\langle y, \sum_{j \neq j_{0}} \mathscr{G}_{j}-\mathscr{D}_{\text {tot }}\right\rangle-\sum_{j \neq j_{0}} \operatorname{deg}\left(\mathcal{G}_{j}\right)+\operatorname{deg} \underset{j \in\{2,3\}}{\operatorname{pgcd}}\left(\mathcal{G}_{j}+\sum_{i \in I_{j}} b_{i, j} \mathcal{D}_{i}\right), \\
\psi_{j_{0}}(y, \mathcal{G}, \mathcal{D}) \stackrel{\text { déf }}{=}\left\langle y, \mathscr{G}_{j_{0}}\right\rangle-\operatorname{deg}\left(\mathcal{G}_{j_{0}}\right)+\operatorname{deg} \underset{j \in J}{\operatorname{pgcd}}\left(\mathcal{G}_{j}+\sum_{i \in I_{j}} b_{i, j} \mathcal{D}_{i}\right)-\operatorname{deg} \underset{j \in J \backslash\left\{j_{0}\right\}}{\operatorname{pgcd}}\left(\mathcal{G}_{j}+\sum_{i \in I_{j}} b_{i, j} \mathcal{D}_{i}\right) \\
\text { et } \Theta(y, \mathcal{G}, \mathcal{D}) \stackrel{\text { déf }}{=} \varphi_{j_{0}}(y, \mathcal{G}, \mathcal{D})+\psi_{j_{0}}(y, \mathcal{G}, \mathcal{D}) .
\end{gathered}
$$

On notera que $\Theta$ ne dépend pas du choix de $j_{0}$. Le lemme suivant découle facilement de [Bou09a, Lemme 3.5 et Corollaire 3.6].

Lemme 5.1. - Soit $y \in \operatorname{Pic}(X)^{\vee} \cap C_{\text {eff }}(X)^{\vee}$, et $(\mathcal{G}, \mathcal{D}) \in \operatorname{Div}_{\text {eff }}(\mathscr{C})^{J \times I}$ vérifiant $\operatorname{deg}\left(\mathcal{D}_{i}\right)=\left\langle y, \mathscr{F}_{i}\right\rangle$ pour $i \in I$.

1. Supposons $\varphi_{j_{0}}(y, \mathcal{G}, \mathcal{D}) \geqslant 2 g_{\mathscr{C}}-1$ et $\psi_{j_{0}}(y, \mathcal{G}, \mathcal{D}) \geqslant 2 g_{\mathscr{C}}-1$. Alors

$$
\mathscr{N}(\mathcal{G}, \mathcal{D})=q^{\left(1-g_{\mathscr{C}}\right) \operatorname{dim}(X)+\Theta(y, \mathcal{G}, \mathcal{D})}
$$

et

$$
\mathscr{N}_{j_{0}}(\mathcal{G}, \mathcal{D})=q^{\left(1-g_{\mathscr{C}}\right) \operatorname{dim}(X)+\varphi_{j_{0}}(y, \mathcal{G}, \mathcal{D})} .
$$

2. Supposons $\varphi_{j_{0}}(y, \mathcal{G}, \mathcal{D}) \geqslant 0$ Alors

$$
\mathscr{N}_{j_{0}}(\mathcal{G}, \mathcal{D}) \leqslant q^{\operatorname{dim}(X)+\varphi_{j_{0}}(y, \mathcal{G}, \mathcal{D})} .
$$

3. Supposons $\psi_{j_{0}}(y, \mathcal{G}, \mathcal{D})<0$. Alors

$$
\mathscr{N}^{*}(\mathcal{G}, \mathcal{D})=0 .
$$

4. Supposons $\varphi_{j_{0}}(y, \mathcal{G}, \mathcal{D}) \leqslant 2 g_{\mathscr{C}}-2$. Alors

$$
\mathscr{N}^{*}(\mathcal{G}, \mathcal{D}) \leqslant q^{\operatorname{dim}(X)+2 g_{\mathscr{G}}-2+\psi_{j_{0}}(\mathcal{G}, \mathcal{D})} .
$$

5. Supposons $\varphi_{j_{0}}(y, \mathcal{G}, \mathcal{D}) \geqslant 0$ et $\psi_{j_{0}}(y, \mathcal{G}, \mathcal{D}) \geqslant 0$. Alors

$$
\mathscr{N}^{*}(\mathcal{G}, \mathcal{D}) \leqslant q^{\operatorname{dim}(X)+\Theta(y, \mathcal{G}, \mathcal{D})} .
$$




\section{Le résultat principal}

Supposons $k$ fini. Soit $f: \operatorname{Pic}(X)^{\vee} \cap C_{\text {eff }}(X)^{\vee} \rightarrow \mathbf{R}$ une fonction. Soit $\mathcal{P}$ une partie de $C_{\text {eff }}(X)^{\vee}$ qui est une union finie de cônes. Pour $\alpha \in\{I, I I\}$, on dit que $f$ est un terme d'erreur de type $\alpha$ sur $\mathcal{P}$ si

- cas $\alpha=I$ : la série

$$
\sum_{y \in \mathcal{P} \cap \operatorname{Pic}(X)^{\vee}} f(y) t^{\left\langle y,-\mathscr{K}_{X}\right\rangle}
$$

est $q^{-1}$-contrôlée à l'ordre $\operatorname{rg}(\operatorname{Pic}(X))-1$.

$-\operatorname{cas} \alpha=I I$ : on a

$$
\lim _{\substack{y \in \mathcal{P} \cap \operatorname{Pic}(X)^{\vee} \\ \operatorname{dist}(y, \partial \mathcal{P}) \rightarrow+\infty}} q^{\left\langle y, \mathscr{K}_{X}\right\rangle} f(y)=0 .
$$

On dit que $f$ est un terme d'erreur de type $\alpha \in\{I, I I\}$ si $f$ est un terme d'erreur de type $\alpha$ sur $C_{\text {eff }}(X)^{\vee}$.

Pour $\alpha \in\{I, I I\}$, on dit que $f$ est un terme dominant de type $\alpha$ sur $\mathcal{P}$ (respectivement un terme dominant de type $\alpha)$ si $f(y)-q^{\left\langle y,-\mathscr{K}_{X}\right\rangle} \gamma(X)$ est un terme d'erreur de type $\alpha$ sur $\mathcal{P}$ (respectivement un terme d'erreur de type $\alpha$ ). On emploiera l'expression « de type I fort » quand la propriété correspondante vaut sur toute partie $\mathcal{P}^{\prime}$ de $\mathcal{P}$ qui est une union finie de cônes.

Remarque 6.1. - Aucune des deux propriétés « $f$ est un terme d'erreur de type $\alpha$ sur $\mathcal{P} »$ pour $\alpha \in\{I$ fort, $I I\}$ n'implique a priori l'autre. Cependant, en supposant que $\mathcal{P}$ est un cône et que $\left\{x_{0}, \ldots, x_{r}\right\}$ sont des éléments non nuls de son dual, elles découlent toutes les deux de la propriété plus forte suivante :

$$
\exists \varepsilon>0, \quad \forall y \in \mathcal{P} \cap \operatorname{Pic}(X)^{\vee}, \quad\left|q^{\left\langle y, \mathscr{K}_{X}\right\rangle} f(y)\right| \ll \sum_{i=0}^{r} q^{-\varepsilon\left\langle y, x_{i}\right\rangle} .
$$

C'est immédiat pour $\alpha=I I$ et pour $\alpha=I$ fort, on utilise le point 1 de [Bou11a, Lemme 13].

Supposons $k=\mathbf{Q}$. Soit, pour presque tout premier $p, f_{p, r}: \operatorname{Pic}(X)^{\vee} \cap C_{\text {eff }}(X)^{\vee} \rightarrow$ $\mathbf{R}$ une famille de fonction indexées par $r \in \mathbf{N}_{>0}$. Soit $\mathcal{P}$ une partie de $C_{\text {eff }}(X)^{\vee}$. On dit que $\left\{f_{p, r}\right\}$ est un terme d'erreur sur $\mathcal{P}$ s'il existe une constante positive $M$ dépendant de $\mathscr{C}, X$, et $\mathcal{P}$ telle que pour tout $y \in C_{\text {eff }}(X)^{\vee} \cap \operatorname{Pic}(X)^{\vee}$ vérifiant $\operatorname{dist}(y, \partial \mathcal{P}) \geqslant M$ on a pour presque tout $p$

$$
\lim _{r \rightarrow+\infty} p^{r\left[\left(g_{\mathscr{C}}-1\right) \operatorname{dim}(X)+\left\langle y, \mathscr{K}_{X}\right\rangle\right]} f_{p, r}(y)=0 .
$$

On dit que $\left\{f_{p, r}\right\}$ est un terme d'erreur si $\left\{f_{p, r}\right\}$ est un terme d'erreur sur $C_{\text {eff }}(X)^{\vee}$. On dit que $\left\{f_{p, r}\right\}$ est un terme dominant sur $\mathcal{P}$ (respectivement un terme dominant) si, pour presque tout $p, f_{p, r}(y)-p^{r\left[\left(1-g_{\mathscr{G}}\right) \operatorname{dim}(X)+\left\langle y,-\mathscr{K}_{X}\right\rangle\right]}$ est un terme d'erreur sur $\mathcal{P}$ (respectivement un terme d'erreur).

On considère toujours une hypersurface intrinsèque linéaire $X$ telle que $\operatorname{dim}(X)=2$ et un ensemble admissible de variables $J$. Soit $y \in \operatorname{Pic}(X)^{\vee} \cap C_{\text {eff }}(X)^{\vee}$. Si $k$ est fini, 
on pose

$$
\begin{gathered}
n_{0}^{J}(y) \stackrel{\text { déf }}{=} \sum^{J} \nu_{X, J} \cdot q^{\left(1-g_{\mathscr{C}}\right) \operatorname{dim}(X)+\Theta} \\
n_{1}^{J}(y) \stackrel{\text { déf }}{=} \sum_{\exists j \in J, \quad \psi_{j}<0}^{y} \nu_{X, J} . q^{\left(1-g_{\mathscr{C}}\right) \operatorname{dim}(X)+\Theta} \\
+\sum_{\substack{\exists \in J, \forall j \in J, \varphi_{j} \geqslant 2 g_{\mathscr{C}} \geqslant 0}}^{y} \nu_{X, J} \cdot\left(q^{\left(1-g_{\mathscr{C}}\right) \operatorname{dim}(X)+\Theta}-\mathscr{N}^{*}\right)
\end{gathered}
$$

et

$$
n_{2}^{J}(y) \stackrel{\text { déf }}{=} \sum_{\substack{\forall j \in J, \forall j \in J, \boldsymbol{c}_{j} \leqslant 2 \phi_{\mathscr{C}}-2}}^{y} \nu_{X, J} \cdot\left(q^{\left(1-g_{\mathscr{C}}\right) \operatorname{dim}(X)+\Theta}-\mathscr{N}^{*}\right) .
$$

Si $k=\mathbf{Q}$, on définit de manière analogue $n_{0, p, r}^{J}(y), n_{1, p, r}^{J}(y)$ et $n_{2, p, r}^{J}(y)$.

Théorème 6.2. - 1. Soit $y \in \operatorname{Pic}(X)^{\vee} \cap C_{\mathrm{eff}}(X)^{\vee}$. Si k est fini, on a

$$
\# \boldsymbol{H o m}_{X_{0}}(\mathscr{C}, X, y)(k)=n_{0}^{J}(y)+n_{1}^{J}(y)+n_{2}^{J}(y) .
$$

Si $k=\mathbf{Q}$, on a pour presque tout $p$ et tout $r \geqslant 1$

$$
\# \boldsymbol{H o m}_{X_{0}}(\mathscr{C}, X, y)\left(\mathbf{F}_{p^{r}}\right)=n_{0, p, r}^{J}(y)+n_{1, p, r}^{J}(y)+n_{2, p, r}^{J}(y) .
$$

2. Pour $\alpha \in\{I$ fort, $I I\}, n_{0}^{J}$ est un terme dominant de type $\alpha ;\left\{n_{0, p, r}^{J}\right\}$ est un terme dominant.

3. Pour $\alpha \in\{I$ fort, $I I\}, n_{0}^{J}$ est un terme d'erreur de type $\alpha ;\left\{n_{1, p, r}^{J}\right\}$ est un terme d'erreur.

4. Soit $\mathcal{C}_{\lambda} \stackrel{\text { déf }}{=}\left\{y \in C_{\text {eff }}(X)^{\vee},\left\langle y,(1-\lambda) \cdot\left(\mathscr{G}_{j_{1}}+\mathscr{G}_{j_{2}}\right)-\mathscr{D}_{\text {tot }}\right\rangle \geqslant 0\right\}$. Pour tout $\lambda>0$, et pour $\alpha \in\{I$ fort, $I I\}, n_{2}^{J}$ est un terme d'erreur de type $\alpha$ sur $\mathcal{C}_{\lambda}$; pour tout $\lambda>0,\left\{n_{2, p, r}^{J}\right\}$ est un terme d'erreur sur $\mathcal{C}_{\lambda}$.

Avant d'en venir à la démonstration de ce théorème, nous en donnons quelques corollaires immédiats.

Corollaire 6.3. - Pour $\alpha \in\{I, I$ fort, $I I\}$, "Manin $\alpha$ »vaut pour $\left(X, X_{0}\right)$ si et seulement si $n_{2}^{J}$ est un terme d'erreur de type $\alpha$. "Manin géométrique » vaut pour $\left(X, X_{0}\right)$ si et seulement si $\left\{n_{2, p, r}^{J}\right\}$ est un terme d'erreur.

Remarque 6.4. - L'étude de $n_{2}^{J}$ dans le présent travail est basée sur la majoration grossière (7.3.1). Il semble clair qu'en général le membre de droite de (7.3.1) ne sera pas un terme d'erreur, et que seule une étude fine de la différence $q^{\left(1-g_{\mathscr{C}}\right)} \operatorname{dim}(X)+\Theta-\mathscr{N}^{*}$ pourra permettre de montrer que $n_{2}^{J}$ est bien un terme d'erreur. 
Pour $\lambda \geqslant 0$, soit

$$
\mathcal{P}_{\lambda}=\cup_{J} \mathcal{C}_{\lambda}, \quad \mathcal{P}_{\lambda}^{\prime}=C_{\text {eff }}(X)^{\vee} \backslash \bigcap_{J}\left\{\left\langle\bullet,(1-\lambda) \cdot\left(\mathscr{G}_{j_{1}}+\mathscr{G}_{j_{2}}\right)-\mathscr{D}_{\text {tot }}\right\rangle \leqslant 0\right\}
$$

où $J \subset \mathfrak{I}$ décrit à chaque fois l'ensemble des choix admissibles de variables, de sorte que $\overline{\mathcal{P}_{\lambda}^{\prime}}=\mathcal{P}_{\lambda}$.

Corollaire 6.5. - Pour tout $\lambda>0$, «Manin I fort "vaut pour $\left(X, X_{0}\right)$ sur $\mathcal{P}_{\lambda}$, et "Manin II » et " Manin géométrique » valent pour $\left(X, X_{0}\right)$ sur $\mathcal{P}_{\lambda}^{\prime}$.

$S^{\prime}$ 'il existe $\lambda>0$ tel que $\mathcal{P}_{\lambda}=C_{\text {eff }}(X)^{\vee}$, "Manin I fort " vaut pour $\left(X, X_{0}\right)$.

$S^{\prime}$ 'il existe $\lambda>0$ tel qu'on ait $\operatorname{int}\left(C_{\mathrm{eff}}(X)^{\vee}\right) \subset \mathcal{P}_{\lambda}^{\prime}$, "Manin II " et "Manin géométrique » valent pour $\left(X, X_{0}\right)$.

Corollaire 6.6. — "Manin I fort », "Manin II ", et " Manin géométrique » valent pour les trois surfaces de del Pezzo généralisées suivantes : la sextique avec singularité $\mathbf{A}_{1}$, la quintique avec singularité $\mathbf{A}_{2}$ et la quartique avec singularités $\mathbf{A}_{3}+\mathbf{A}_{1}$. Pour les cinq surfaces de del Pezzo généralisées suivantes : la sextique avec singularité $\mathbf{A}_{2}$, les quintiques avec singularités respectives $\mathbf{A}_{1}$ et $\mathbf{A}_{2}$, les quartiques avec singularités respectives $3 \mathbf{A}_{1}, \mathbf{A}_{2}+\mathbf{A}_{1}$, et $\mathbf{A}_{3}$, on a

$$
\limsup _{\mathcal{P}, \text { Manin vaut sur } \mathcal{P}} \frac{\operatorname{Vol}(\mathcal{P})}{\operatorname{Vol}\left(C_{\text {eff }}(X)^{\vee}\right)}=1 .
$$

Le dernier corollaire découle aussitôt du corollaire précédent et d'un calcul Maple utilisant [Fra09] et les données fournies par [Der06]. Pour toutes ces surfaces, on vérifie en effet qu'on a $\mathcal{P}_{0}=C_{\text {eff }}(X)^{\vee}$ et pour les trois premières on a $\mathcal{P}_{\lambda}^{\prime} \cup\{0\}=$ $C_{\text {eff }}(X)^{\vee}$ pour $\lambda>0$ assez petit.

On obtient d'ailleurs en toute généralités les résultats numérique présentés dans la table 1 pour les 20 surfaces de [Der06] qui sont des hypersurfaces linéaires intrinsèques. On notera en particulier que les résultats présentés dans la table 1 de [Bou11b] sont sensiblement améliorés.

Remarque 6.7. - En employant des techniques strictement similaires à celles utilisées ici, on pourrait en fait montrer qu'on peut remplacer dans les résultats cidessus l'ouvert $X_{0}$ par $X$. En particulier, on obtiendrait pour les degrés $y$ ad hoc que $\operatorname{Hom}_{X_{0}}(\mathscr{C}, X, y)$ est dense dans $\operatorname{Hom}(\mathscr{C}, X, y)$. Les détails seraient cependant assez laborieux ( $c f$. [Bou11b] où ce type d'énoncé est considéré).

Passons à la démonstration du théorème 6.2. Le premier point du théorème découle aussitôt de (3.4.6), (3.4.7) et des définitions. La démonstration des autres points occupe la section suivante. 


\begin{tabular}{|c|c|c|}
\hline degré & singularités & $\lim _{\sup } \mathcal{P}$, Manin vaut sur $\mathcal{P} \frac{\operatorname{Vol}(\mathcal{P})}{\operatorname{Vol}\left(C_{\text {eff }}(X)^{\vee}\right)}$ \\
\hline 6 & $\overline{\mathbf{A}_{1}}$ & 1 \\
\hline 6 & $\mathbf{A}_{2}$ & 1 \\
\hline 5 & $\mathbf{A}_{1}$ & 1 \\
\hline 5 & $\mathbf{A}_{2}$ & 1 \\
\hline 5 & $\mathbf{A}_{3}$ & $\geqslant 0.81$ \\
\hline 5 & $\mathbf{A}_{4}$ & $\geqslant 0.76$ \\
\hline 4 & $3 \mathbf{A}_{1}$ & 1 \\
\hline 4 & $\mathbf{A}_{2}+\mathbf{A}_{1}$ & 1 \\
\hline 4 & $\mathbf{A}_{3}+\mathbf{A}_{1}$ & 1 \\
\hline 4 & $\mathbf{A}_{3}$ & 1 \\
\hline 4 & $\mathbf{A}_{4}$ & $\geqslant 0.72$ \\
\hline 4 & $\mathbf{D}_{4}$ & $\geqslant 0.43$ \\
\hline 4 & $\mathbf{D}_{5}$ & $\geqslant 0.34$ \\
\hline 3 & $2 \mathbf{A}_{2}+\mathbf{A}_{1}$ & $\geqslant 0.97$ \\
\hline 3 & $\mathbf{A}_{3}+2 \mathbf{A}_{1}$ & $\geqslant 0.92$ \\
\hline 3 & $\mathbf{A}_{4}+\mathbf{A}_{1}$ & $\geqslant 0.98$ \\
\hline 3 & $\mathbf{A}_{5}+\mathbf{A}_{1}$ & $\geqslant 0.68$ \\
\hline 3 & $\mathbf{D}_{4}$ & $\geqslant 0.95$ \\
\hline 3 & $\mathbf{D}_{5}$ & $\geqslant 0.28$ \\
\hline 3 & $\mathbf{E}_{6}$ & $\geqslant 0.08$ \\
\hline
\end{tabular}

TABLE 1

\section{Démonstration du théorème 6.2}

7.1. Démonstration du point 2 du théorème 6.2. - Supposons $k$ fini. D'après (6.0.91), (4.0.37), (5.0.80) et (3.2.2), on a

$$
q^{\left(g_{\mathscr{C}}-1\right) \operatorname{dim}(X)+\left\langle y, \mathscr{K}_{X}\right\rangle} n_{0}^{J}(y)=\sum_{\substack{\mathcal{S} \in \operatorname{Div}_{\text {eff }}(\mathscr{C})^{J} \\ \operatorname{deg}\left(\mathscr{\mathcal { G }}_{j}\right) \leqslant\left\langle y, \mathscr{G}_{j}\right\rangle,}} q^{-\sum_{i \in I}\left\langle y, \mathscr{F}_{i}\right\rangle} \mathscr{M}\left(\left(\left\langle y, \mathscr{F}_{i}\right\rangle\right), \mathcal{G}\right) q^{-|\operatorname{deg}(\boldsymbol{G})|}
$$

et donc d'après le point 5 de la proposition 4.6 on a pour tout $\varepsilon>0$ assez petit

$$
\begin{aligned}
& \quad\left|q^{\left\langle y, \mathscr{K}_{X}\right\rangle} n_{0}^{J}(y)-\gamma(X)\right| \\
& \ll_{\varepsilon} \sum_{i \in I} q^{-\varepsilon\left\langle y, \mathscr{F}_{i}\right\rangle} \sum_{\mathcal{G} \in \operatorname{Div}_{\text {eff }}(\mathscr{C})_{\leqslant 1}^{J}}\left(\prod_{v, v(\mathcal{G}) \neq 0} C \cdot q_{v}^{|v(\mathcal{G})|-2+\varepsilon}\right) q^{-|\operatorname{deg}(\mathcal{G})|} \\
& \quad+q^{-\varepsilon \sum_{j \in J}\left\langle y, \mathscr{G}_{j}\right\rangle} \\
& \ll_{\varepsilon} q^{-\varepsilon \sum_{j \in J}\left\langle y, \mathscr{G}_{j}\right\rangle}+\sum_{i \in I} q^{-\varepsilon\left\langle y, \mathscr{F}_{i}\right\rangle} .
\end{aligned}
$$


Ceci montre le résultat pour $n_{0}^{J}$.

Supposons à présent $k=\mathbf{Q}$. On a

$$
p^{r\left[\left(g_{\mathscr{C}}-1\right) \operatorname{dim}(X)+\left\langle y, \mathscr{K}_{X}\right\rangle\right]} n_{0, p, r}^{J}(y)=\sum_{\substack{\mathcal{G} \in \operatorname{Div}_{\text {eff }}\left(\mathscr{C}_{p, r}\right)^{J} \\ \operatorname{deg}\left(\mathcal{G}_{j}\right) \leqslant\left\langle y, \mathscr{G}_{j}\right\rangle, \quad j \in J}} p^{-r \sum_{i \in I}\left\langle y, \mathscr{F}_{i}\right\rangle} \mathscr{M}_{p, r}\left(\left(\left\langle y, \mathscr{F}_{i}\right\rangle\right), \mathcal{G}\right) p^{-r|\operatorname{deg}(\mathcal{G})|}
$$

et donc d'après le point 6 de la proposition 4.6 , pour tout $\varepsilon>0$ assez petit,

$$
\left|p^{r\left[\left(g_{\mathscr{C}}-1\right) \operatorname{dim}(X)+\left\langle y, \mathscr{K}_{X}\right\rangle\right]} n_{0, p, r}^{J}(y)-c(p, r)\right|
$$

est majoré par

$$
C(p, r, \varepsilon)\left[\sum_{i \in I} p^{-r \varepsilon\left\langle y, \mathscr{F}_{i}\right\rangle}+\sum_{\substack{\mathcal{G} \in \operatorname{Div}_{\text {eff }}\left(\mathscr{C}_{p, r}\right)_{\leqslant 1}^{J} \\ \mathcal{G} \neq(0, \ldots, 0)}}\left(\prod_{v, v(\mathcal{G}) \neq 0} C \cdot p_{v}^{r|v(\mathcal{G})|-2+\varepsilon}\right) p^{-r|\operatorname{deg}(\mathbf{S})|} .\right]
$$

avec $\lim _{r \rightarrow+\infty} C(p, r, \varepsilon)=1$. D'après [Bou11b, Lemma 5.2], la dernière somme tend vers 0 quand $r \rightarrow+\infty$. On en déduit le résultat pour $n_{0, p, r}^{J}$.

7.2. Démonstration du point $3 \mathrm{du}$ théorème 6.2. — Supposons $k$ fini. On a

$$
\left|n_{1}^{J}(y)\right| \ll \sum_{\exists j \in J, \psi_{j}<0}^{y}\left|\nu_{X, J}\right| \cdot q^{\Theta}+\sum_{j_{0} \in J} \sum_{\substack{\varphi_{j_{0}} \geqslant 2 g_{\mathscr{C}}-1 \\ \forall j \in J, \psi_{j} \geqslant 0}}^{y}\left|\nu_{X, J}\right| \cdot\left|q^{\left(g_{\mathscr{C}}-1\right) \operatorname{dim}(X)+\Theta}-\mathscr{N}^{*}\right|
$$

et pour tout $\eta \leqslant 1$

$$
\sum_{\exists j \in J, \psi_{j}<0}^{y}\left|\nu_{X, J}\right| \cdot q^{\Theta} \ll \sum_{j_{0} \in J} \sum^{y}\left|\nu_{X, J}\right| \cdot q^{\varphi_{j_{0}}+(1-\eta) \psi_{j_{0}}} .
$$

Par ailleurs, d'après le point $1 \mathrm{du}$ lemme 5.1 , on a

$$
\begin{aligned}
& \sum_{\substack{\varphi_{j} \geqslant 2 g_{\mathscr{C}}-1 \\
\forall j \in J, \psi_{j} \geqslant 0}}^{y}\left|\nu_{X, J}\right| \cdot\left|q^{\left(g_{\mathscr{C}}-1\right) \operatorname{dim}(X)+\Theta}-\mathscr{N}^{*}\right| \\
= & \sum_{\substack{\varphi_{j_{0}} \geqslant 2 g_{\mathscr{C}}-1 \\
\forall j \in J, \psi_{j} \geqslant 0 \\
\psi_{j_{0}} \geqslant 2 g_{\mathscr{C}}-1}}^{y}\left|\nu_{X, J}\right| \cdot\left|-1+\sum_{j \in J} \mathscr{N}_{j}\right|+\sum_{\substack{\varphi_{j_{0}} \geqslant 2 g_{\mathscr{C}}-1 \\
0 \leqslant \psi_{j_{0}} \leqslant 2 g_{\mathscr{C}}-2}}^{y}\left|\nu_{X, J}\right|\left|q^{\left(1-g_{\mathscr{C}}\right) \operatorname{dim}(X)+\Theta}-\mathscr{N}^{*}\right| .
\end{aligned}
$$


En utilisant le point 2 du lemme 5.1 on obtient pour tout $\eta \leqslant 1$

$$
\begin{aligned}
& \sum_{\substack{\varphi_{j_{0}} \geqslant 2 g_{\mathscr{C}}-1 \\
\forall j \in J, \psi_{j} \geqslant 0 \\
\psi_{j_{0}} \geqslant 0}}^{y}\left|\nu_{X, J}\right| \cdot\left|-1+\sum_{j \in J} \mathscr{N}_{j}\right| \ll \sum_{j \in J} \sum_{\forall j \in J, \psi_{j} \geqslant 0}^{y}\left|\nu_{X, J}\right| q^{\varphi_{j_{0}}} \\
& \ll \sum_{j \in J} \sum^{y}\left|\nu_{X, J}\right| q^{\varphi_{j_{0}}+(1-\eta) \psi_{j_{0}}} .
\end{aligned}
$$

Finalement, en utilisant le point $5 \mathrm{du}$ lemme 5.1 on obtient pour $\eta \geqslant 0$

$$
\sum_{\substack{\varphi_{j_{0}} \geqslant 2 g_{\mathscr{C}}-1 \\ 0 \leqslant \psi_{j_{0}} \leqslant 2 g_{\mathscr{C}}-2}}^{y}\left|\nu_{X, J}\right|\left|q^{\left(1-g_{\mathscr{C}}\right) \operatorname{dim}(X)+\Theta}-\mathscr{N}^{*}\right| \ll \eta \sum^{y}\left|\nu_{X, J}\right| q^{\varphi_{j_{0}}+(1-\eta) \psi_{j_{0}}}
$$

Pour $0 \leqslant \eta \leqslant 1$ on obtient en définitive

$$
\left|n_{1}^{J}(y)\right| \ll_{\eta} \sum_{j \in J} \sum^{y}\left|\nu_{X, J}\right| \cdot q^{\varphi_{j}+(1-\eta) \psi_{j}}
$$

Notons que d'après $(4.0 .38),(5.0 .79),(5.0 .78)$ et $(3.2 .2)$ on a pour tout $\eta$ et tout $j \in J$

$$
\begin{aligned}
& q^{\left\langle y, \mathscr{K}_{X}\right\rangle} \sum^{y}\left|\nu_{X, J}\right| \cdot q^{\varphi_{j}+(1-\eta) \psi_{j}} \\
= & q^{-\eta\left\langle y, \mathscr{G}_{j}\right\rangle} \sum_{\mathcal{G} \in \operatorname{Div}_{\mathrm{eff}}(\mathscr{C})_{\leqslant 1}^{J}} q^{-\sum_{i \in I}\left\langle y, \mathscr{F}_{i}\right\rangle} \mathscr{M}_{j, \eta}\left(\left\langle y, \mathscr{F}_{i}\right\rangle, \mathcal{G}\right) q^{-|\operatorname{deg}(\mathcal{G})|+\eta \operatorname{deg}\left(\mathcal{G}_{j}\right)}
\end{aligned}
$$

En appliquant le point 5 de la proposition 4.6, pour tout $\varepsilon>0$ assez petit, on obtient pour tout $\eta>0$ assez petit la majoration

$$
q^{\left\langle y, \mathscr{K}_{X}\right\rangle}\left|n_{1}^{J}(y)\right| \ll_{\varepsilon, \eta} \sum_{j \in J} q^{-\eta\left\langle y, \mathscr{G}_{j}\right\rangle}
$$

d'où le résultat.

Supposons $k=\mathbf{Q}$. Par un raisonnement analogue à ci-dessus, on a pour tout $\eta$

$$
\left|n_{1, p, r}^{J}(y)\right| \ll_{\eta} p^{r\left(\operatorname{dim}(X)+\eta\left(2 g_{\mathscr{C}}-2\right)\right)} \sum_{j \in J} \sum^{y}\left|\nu_{X, J, p, r}\right| \cdot p^{r\left(\varphi_{j}+(1-\eta) \psi_{j}\right)}
$$


Pour tout $j \in J$ et tout $\varepsilon>0$ assez petit, en appliquant le point 6 de la proposition 4.6 , on obtient pour $\eta>0$ assez petit

$$
\begin{aligned}
p^{r\left(\left(g_{\mathscr{C}}-1\right) \operatorname{dim}(X)+\left\langle y, \mathscr{K}_{X}\right\rangle\right)} p^{r\left(\operatorname{dim}(X)+\eta\left(2 g_{\mathscr{C}}-2\right)\right)} \sum^{y}\left|\nu_{X, J, p, r}\right| \cdot p^{r\left(\varphi_{j}+(1-\eta) \psi_{j}\right)} \\
=p^{r\left(g_{\mathscr{C}} \operatorname{dim}(X)+\eta\left(2 g_{\mathscr{C}}-2\right)-\eta\left\langle y, \mathscr{G}_{j}\right\rangle\right)} p^{-r \sum_{i \in I}\left\langle y, \mathscr{F}_{i}\right\rangle} \\
\quad \times \sum_{\mathcal{G} \in \operatorname{Div}_{\mathrm{eff}}\left(\mathscr{C}_{p, r}\right)_{\leqslant 1}^{J}} \mathscr{M}_{j, \eta, p, r}\left(\left\langle y, \mathscr{F}_{i}\right\rangle, \mathcal{G}\right) p^{-r\left(|\operatorname{deg}(\mathcal{G})|+\eta \operatorname{deg}\left(\mathcal{G}_{j}\right)\right)} \\
\leqslant C(p, r, \varepsilon, \eta) p^{r\left(g_{\mathscr{C}} \operatorname{dim}(X)+\eta\left(2 g_{\mathscr{C}}-2\right)-\eta\left\langle y, \mathscr{G}_{j}\right\rangle\right)}
\end{aligned}
$$

avec $\lim _{r \rightarrow+\infty} C(p, r, \varepsilon, \eta)=1$ (on a encore utilisé [Bou11b, Lemma 5.2]) d'où le résultat (rappelons que l'on peut exprimer explicitement un $\eta$ convenable en fonction de $X)$.

7.3. Démonstration du point 4 du théorème 6.2. - Supposons $k$ fini. Écrivons $J=\left\{j_{0}, j_{1}, j_{2}\right\}$. D'après les points 4 et $5 \mathrm{du}$ lemme 5.1 , on a la majoration

$$
\left|n_{2}^{J}(y)\right| \ll \sum^{y}\left|\nu_{X, J}\right| \cdot q^{\psi_{j_{0}}} .
$$

Soit $y$ vérifiant $\left\langle y, \mathscr{G}_{j}\right\rangle \leqslant \operatorname{deg}\left(\mathcal{G}_{j}\right)$ pour tout $j \in J$. D'après (5.0.79), on a pour $\lambda>0$

$$
\begin{gathered}
\psi_{j_{0}}(y, \mathcal{G}, \mathcal{D}) \leqslant\left\langle y, \sum_{j \in J} \mathscr{G}_{j}-\mathscr{D}_{\text {tot }}\right\rangle-\left\langle y,(1-\lambda) \mathscr{G}_{j_{1}}+\mathscr{G}_{j_{2}}-\mathscr{D}_{\text {tot }}\right\rangle-\operatorname{deg}\left(\mathcal{G}_{1}\right) \\
\quad+\operatorname{deg} \underset{j \in J}{\operatorname{pgcd}}\left(\mathcal{G}_{j}+\sum_{i \in I_{j}} b_{i, j} \mathcal{D}_{i}\right)-\lambda\left(\operatorname{deg}\left(\mathcal{G}_{j_{1}}\right)+\operatorname{deg}\left(\mathcal{G}_{j_{2}}\right)\right)-\operatorname{deg}\left(\operatorname{pgcd}\left(\mathcal{G}_{j_{1}}, \mathcal{G}_{j_{2}}\right)\right) .
\end{gathered}
$$

En utilisant (4.0.49), on obtient pour tout $\varepsilon>0$ assez petit la majoration

$$
\begin{aligned}
& q^{\left\langle y, \mathscr{K}_{X}\right\rangle} \sum^{y}\left|\nu_{X, J}\right| \cdot q^{\psi_{j_{0}}} \\
& \ll_{\varepsilon} \sum_{\mathcal{G} \in \operatorname{Div}_{\mathrm{eff}}(\mathscr{C})_{\leqslant 1}^{J}}\left(\prod_{v, v(\mathcal{G}) \neq 0} C \cdot q_{v}^{|v(\mathcal{G})|-2+\varepsilon}\right) q^{-\operatorname{deg}\left(\mathcal{G}_{j_{0}}\right)-\operatorname{deg}\left(\operatorname{pgcd}\left(\mathcal{G}_{j_{1}}, \mathcal{G}_{j_{2}}\right)\right)-\lambda\left(\operatorname{deg}\left(\mathcal{S}_{j_{1}}\right)-\operatorname{deg}\left(\mathcal{G}_{j_{2}}\right)\right)} \\
& \times q^{-\left\langle y,(1-\lambda) \mathscr{G}_{j_{1}}+\mathscr{G}_{j_{2}}-\mathscr{D}_{\text {tot }}\right\rangle}
\end{aligned}
$$

On vérifie aussitôt qu'on a pour $\boldsymbol{g} \in\{0,1\}^{J}$ et $\varepsilon>0$ assez petit

$$
|\boldsymbol{g}|-2+\varepsilon-g_{j_{0}}-\operatorname{Min}\left(g_{j_{1}}, g_{j_{2}}\right)-\lambda\left(g_{j_{1}}+g_{j_{2}}\right)<-1
$$

d'où la convergence de la série apparaissant dans (7.3.3) et la majoration

$$
q^{\left\langle y, \mathscr{K}_{X}\right\rangle}\left|n_{2}^{J}(y)\right| \leqslant \varepsilon, \lambda q^{-\left\langle y,(1-\lambda) \mathscr{G}_{2}+\mathscr{G}_{3}-\mathscr{D}_{\text {tot }}\right\rangle} .
$$


Si $k=\mathbf{Q}$, on a de même la majoration

$$
p^{r\left[\left(1-g_{\mathscr{C}}\right) \operatorname{dim}(X)\right]}\left|n_{2, p, r}^{J}(y)\right| \ll p^{r\left(g_{\mathscr{C}} \operatorname{dim}(X)+2 g_{\mathscr{C}}-2\right)} \sum^{y}\left|\nu_{X, J, p, r}\right| \cdot p^{r \psi_{j_{0}}} .
$$

et un raisonnement analogue permet de conclure.

\section{Appendice : démonstration du quatrième point de la proposition 4.6}

On commence par remarquer que $F_{j_{0}, 2, \boldsymbol{g}}(\rho, \tau, \boldsymbol{t})$ est la somme des trois séries suivantes :

$$
\begin{aligned}
& \left|\nu_{X, J}^{\circ}(\boldsymbol{g}, \boldsymbol{f})\right|\left(\tau^{j \in J \backslash\left\{j_{0}\right\}} \operatorname{Min}_{j}\left(g_{j}+\sum_{i \in I_{j}} a_{i} f_{i}\right)-\tau\right) \boldsymbol{t}^{\boldsymbol{f}}, \\
& (0, \boldsymbol{f}) \in \mathbf{N}_{\mathrm{inc}}^{\mathfrak{\Im}} \\
& \operatorname{Min}_{j \in J \backslash\left\{j_{0}\right\}}\left(g_{j}+\sum_{i \in I_{j}} a_{i} f_{i}\right) \geqslant 2 \\
& g_{j_{0}}+\sum_{i \in I_{j_{0}}} a_{i} f_{i}=0 \\
& \sum_{\boldsymbol{f} \in \mathbf{N}^{I}}\left|\nu_{X, J}^{\circ}(\boldsymbol{g}, \boldsymbol{f})\right|\left(\rho \tau^{\underset{j \in J \backslash\left\{j_{0}\right\}}{\operatorname{Min}}\left(g_{j}+\sum_{i \in I_{j}} a_{i} f_{i}\right)}-\rho \tau\right) \boldsymbol{t}^{\boldsymbol{f}} \\
& (0, \boldsymbol{f}) \in \mathbf{N}_{\mathrm{in}}^{\mathfrak{\jmath}} \\
& \operatorname{Min}_{j \in J \backslash\left\{j_{0}\right\}}\left(g_{j}+\sum_{i \in I_{j}} a_{i} f_{i}\right) \geqslant 2 \\
& g_{j_{0}}+\sum_{i \in I_{j_{0}}} a_{i} f_{i}=1
\end{aligned}
$$

et

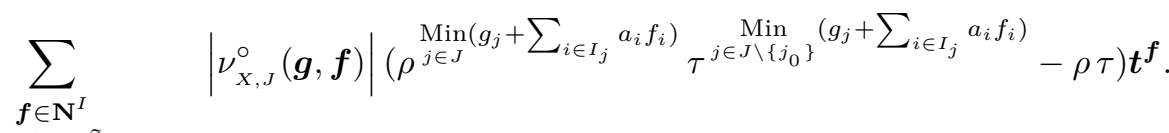

$$
\begin{aligned}
& (0, \boldsymbol{f}) \in \mathbf{N}_{\mathrm{inc}}^{\mathfrak{\Im}} \\
& \operatorname{Min}_{j \in J}\left(g_{j}+\sum_{i \in I_{j}} a_{i} f_{i}\right) \geqslant 2
\end{aligned}
$$

Il s'agit de montrer que ces trois séries sont $|\boldsymbol{g}|$-contrôlées.

8.0.0.1. Le cas de (8.0.7). - On peut bien entendu supposer qu'on a $g_{j_{0}}=0$ Soit $\boldsymbol{f} \in \mathbf{N}^{I}$ vérifiant les conditions sous le signe somme dans (8.0.7). D'après l'hypothèse (4.4), on est alors nécessairement dans l'une des deux situations suivantes :

- il existe $K \in \mathcal{C}_{\text {inc }}$ de cardinal \#J-1 tel que $K \cap I_{j}$ est un singleton pour tout $j \in J \backslash\left\{j_{0}\right\}$ et $f_{i} \geqslant 1$ si et seulement si $i \in K$ ou $i \notin \bigsqcup_{j \in J} I_{j}$ et $\{i\} \cup K \in \mathcal{C}_{\text {inc }}$;

- il existe $K \in \mathcal{C}_{\text {inc }}$ de cardinal \#J et $j_{1} \in J \backslash\left\{j_{0}\right\}$ tel que $\#\left(K \cap I_{j_{1}}\right)=2$ (posons alors $\left.\left\{i_{1}, i_{2}\right\} \stackrel{\text { déf }}{=} K \cap I_{j_{1}}\right), K \cap I_{j}$ est un singleton pour $j \notin\left\{j_{1}, j_{0}\right\}$ et $f_{i}=1$ si et seulement si $i \in K$. 
(8.0.7) se réécrit ainsi comme une somme sur tous les ensembles $K$ du premier type des séries

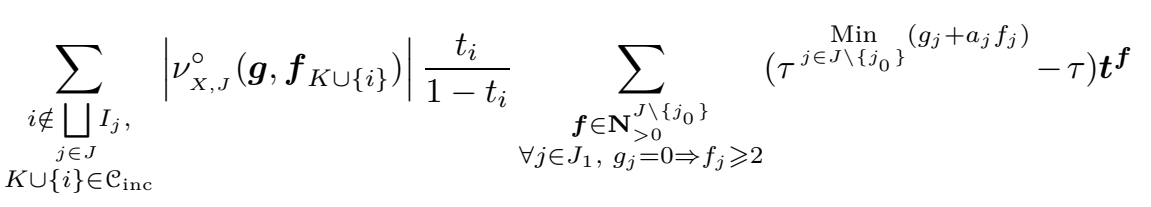

et

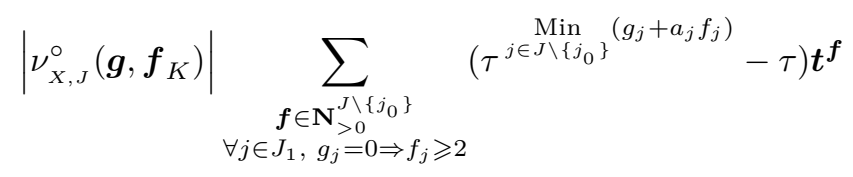

(on a identifié $K$ à $J \backslash\left\{j_{0}\right\}$ et noté $J_{1}=\left\{j \in J \backslash\left\{j_{0}\right\}, a_{j}=1\right\}$ ), plus une somme sur tous les ensembles $K$ du second type des séries

$$
\left|\nu_{X, J}^{\circ}\left(\boldsymbol{g}, \boldsymbol{f}_{K}\right)\right| \sum_{\substack{\boldsymbol{f} \in \mathbf{N}_{>0}^{\left.J \backslash j_{0}, j_{1}\right\}} \\ \forall j \in J_{1}, g_{j}=0 \Rightarrow f_{j} \geqslant 2}}\left(\tau^{\operatorname{Min}\left(g_{j_{1}}+a_{i_{1}} f_{i_{1}}+a_{i_{2}} f_{i_{2}},\left(g_{j}+a_{j} f_{j}\right)_{j \in J \backslash\left\{j_{0}, j_{1}\right\}}\right)}-\tau\right) \boldsymbol{t}^{\boldsymbol{f}}
$$

(on a identifié $K \backslash\left\{i_{1}, i_{2}\right\}$ à $J \backslash\left\{j_{0}, j_{1}\right\}$ et noté $J_{1}=\left\{j \in J \backslash\left\{j_{0}, j_{1}\right\}, a_{j}=1\right\}$ ).

D'après la proposition 4.1, la somme sur $\boldsymbol{f} \in \mathbf{N}_{>0}^{J \backslash\left\{j_{0}\right\}}$ dans (8.0.10) et (8.0.11) vaut, en notant $m$ le plus petit commun multiple des $\left(a_{j}\right)_{j \in J \backslash\left\{j_{0}\right\}}$,

$$
\frac{\tau \prod_{j \in J_{1}} t_{j}^{2-g_{j}} \prod_{j \in J \backslash\left(\left\{j_{0}\right\} \cup J_{1}\right)} t_{j}}{\prod_{j \in J \backslash\left\{j_{0}\right\}}\left(1-t_{j}\right)}\left[\frac{\tau \widetilde{\mathcal{F}}\left(\tau,\left(a_{j}\right)_{j \in J \backslash\left\{j_{0}\right\}}, \boldsymbol{\nu}, \boldsymbol{t}\right)}{\left(1-\tau^{m} \prod_{j \in J \backslash\left\{j_{0}\right\}} t_{j}^{\frac{m}{a_{j}}}\right)}-1\right]
$$

avec $\boldsymbol{\nu}=\left((0, \ldots, 0)_{j \in J_{1}},\left(g_{j}+a_{j}-2\right)_{j \in J \backslash\left(\left\{j_{0}\right\} \cup J_{1}\right)}\right)$.

Toujours d'après la proposition 4.1, la somme sur $\boldsymbol{f} \in \mathbf{N}_{>0}^{J \backslash\left\{j_{0}, j_{1}\right\}}$ dans (8.0.12) vaut, en notant $m_{1}$ le plus petit commun multiple des $\left\{a_{i_{1}},\left(a_{j}\right)_{j \in J \backslash\left\{j_{0}, j_{1}\right\}}\right\}$, et $m_{2}$ le plus petit commun multiple des $\left\{a_{i_{2}},\left(a_{j}\right)_{j \in J \backslash\left\{j_{0}, j_{1}\right\}}\right\}$,

$$
\frac{\tau t_{i_{1}} t_{i_{2}} \prod_{j \in J_{1}} t_{j}^{2-g_{j}} \prod_{j \in J \backslash\left(J_{1} \cup\left\{j_{0}, j_{1}\right)\right\}} t_{j}}{\left(1-t_{i_{1}}\right)\left(1-t_{i_{2}}\right) \prod_{j \in J \backslash\left\{j_{0}, j_{1}\right\}}\left(1-t_{j}\right)}\left[\frac{\rho \widetilde{\mathcal{F}}\left(\tau,\left(a_{i_{1}}, a_{i_{2}},\left\{a_{j}\right\}_{j \in J \backslash\left\{j_{0}, j_{1}\right\}}\right), \boldsymbol{\nu}, \boldsymbol{t}\right)}{\left(1-\tau^{m_{1}} t_{i_{1}{ }^{\frac{m_{1}}{a_{1}}}}^{\prod_{j \in J \backslash\left\{j_{0}, j_{1}\right\}}} t_{j}^{\frac{m_{1}}{a_{j}}}\right)\left(1-\tau^{m_{2}} t_{i_{2}{ }_{j}}^{a_{j \in J}} \prod_{\left.j j_{0}, j_{1}\right\}} t_{j}^{\frac{m_{2}}{a_{j}}}\right)}-1\right]
$$

avec $\boldsymbol{\nu}=\left((0, \ldots, 0)_{j \in J_{1}},\left(g_{j}+a_{j}-2\right)_{j \in J \backslash\left(J_{1} \cup\left\{j_{0}, j_{1}\right\}\right)},\left(g_{j_{1}}+a_{i_{1}}+a_{i_{2}}-2\right)\right)$. Compte tenu de $\# J \geqslant 3$ et de la proposition 4.1 , on vérifie facilement que (8.0.13) et (8.0.14) sont $|\boldsymbol{g}|$-contrôlée. 
8.0.0.2. Le cas de (8.0.9). — En raisonnant comme pour le deuxième point de la proposition 4.6, on voit qu'il suffit de montrer que pour tout $K \in \mathscr{I}$ la série

$$
\left|\nu_{X, J}^{\circ}\left(\boldsymbol{g}, \boldsymbol{f}_{K}\right)\right| \sum_{\substack{\boldsymbol{f} \in \mathbf{N}_{>0}^{J} \\ \forall j \in J_{1}, g_{j}=0 \Rightarrow f_{j} \geqslant 2}}\left(\rho^{\operatorname{Min}\left(g_{j}+a_{j} f_{j}\right)} \tau^{\varepsilon \operatorname{Min}_{j \in J \backslash\left\{j_{0}\right\}}\left(g_{j}+a_{j} f_{j}\right)}-\rho \tau\right) \boldsymbol{t}^{\boldsymbol{f}}
$$

(on a identifié $K$ à $J$ et posé $J_{1} \stackrel{\text { déf }}{=}\left\{j \in J, a_{j}=1\right\}$ ) est $|\boldsymbol{g}|$-contrôlée. Rappelons que par hypothèse $J_{1}$ est non vide. Notons $m$ le plus petit commun multiple de la famille $\left(a_{j}\right)_{j \in J}$ et $n$ le plus petit commun multiple de la famille $\left(a_{j}\right)_{j \in J \backslash\left\{j_{0}\right\}}$. D'après la proposition 4.1, (8.0.15) s'écrit, modulo le facteur $\nu_{X, J}^{\circ}$,

$$
\frac{\rho \tau \prod_{j \in J_{1}} t_{j}^{2-g_{j}} \prod_{j \notin J \backslash J_{1}} t_{j}}{\prod_{j \in J}\left(1-t_{j}\right)}\left[\frac{\tau \widetilde{\mathcal{G}}\left(j_{0}, \rho, \tau,\left(a_{j}\right)_{j \in J}, \boldsymbol{\nu}, \boldsymbol{t}\right)}{\left(1-\rho^{m} \tau^{n} \prod_{j \in J} t_{j}^{\frac{m}{a_{j}}}\right)\left(1-\tau^{n} \prod_{j \in J \backslash\left\{j_{0}\right\}} t_{j}^{\frac{n}{a_{j}}}\right)}-1\right]
$$

avec $\boldsymbol{\nu}=\left((0, \ldots, 0)_{j \in J_{1}},\left(g_{j}+a_{j}-2\right)_{j \notin J_{1}}\right)$. D'après la proposition 4.1, on voit facilement que (8.0.16) est $|\boldsymbol{g}|$-contrôlée.

8.0.0.3. Le cas de (8.0.8). — Dans le cas où $g_{j_{0}}=1$, on se ramène facilement au cas de (8.0.7). Dans le cas où $g_{j_{0}}=0$ on voit qu'il suffit de montrer que pour tout $K \in \mathscr{I}$ contenant $j_{0}$ la série

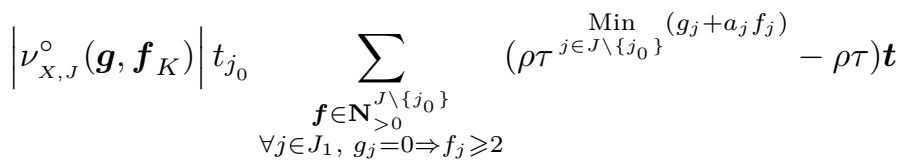

(on a identifié $K$ à $J$ et posé $J_{1} \stackrel{\text { déf }}{=}\left\{j \in J \backslash\left\{j_{0}\right\}, a_{j}=1\right\}$ ) est $|\boldsymbol{g}|$-contrôlée. Comme précédemment, ceci découle aisément d'une application de la proposition 4.1.

\section{Références}

[BB07] R. de la Bretèche \& T. D. Browning. On Manin's conjecture for singular del Pezzo surfaces of degree 4. I. Michigan Math. J., 55(1) :51-80, 2007.

[BBD07] Régis de la Bretèche, Tim D. Browning \& Ulrich Derenthal. On Manin's conjecture for a certain singular cubic surface. Ann. Sci. École Norm. Sup. (4), 40(1) :1-50, 2007.

[BD09a] Tim D. Browning \& Ulrich Derenthal. Manin's conjecture for a quartic del Pezzo surface with $A_{4}$ singularity. Ann. Inst. Fourier (Grenoble), 59(3) :1231-1265, 2009.

[BD09b] Timothy Daniel Browning \& Ulrich Derenthal. Manin's conjecture for a cubic surface with $D_{5}$ singularity. Int. Math. Res. Not. IMRN, (14) :2620-2647, 2009.

[BH07] Florian Berchtold \& Jürgen Hausen. Cox rings and combinatorics. Trans. Amer. Math. Soc., 359(3) :1205-1252 (electronic), 2007.

[Bou09a] David Bourqui. Comptage de courbes sur le plan projectif éclaté en trois points alignés. Ann. Inst. Fourier (Grenoble), 59(5) :1847-1895, 2009. 
[Bou09b] David Bourqui. Produit eulérien motivique et courbes rationnelles sur les variétés toriques. Compos. Math., 145(6) :1360-1400, 2009.

[Bou11a] David Bourqui. La conjecture de Manin géométrique pour une famille de quadriques intrinsèques. Manuscr. Math., 135(1-2) :1-41, 2011.

[Bou11b] David Bourqui. Moduli spaces of curves and Cox rings. à paraître au Michigan Math. J., 2011.

[Deb01] Olivier Debarre. Higher-dimensional algebraic geometry. Universitext. SpringerVerlag, New York, 2001.

[Der06] Ulrich Derenthal. Singular Del Pezzo surfaces whose universal torsors are hypersurfaces. arXiv:math/0604194v1, 2006.

[Der09] Ulrich Derenthal. Counting integral points on universal torsors. Int. Math. Res. Not. IMRN, (14):2648-2699, 2009.

[DT07] Ulrich Derenthal \& Yuri Tschinkel. Universal torsors over del Pezzo surfaces and rational points. In Equidistribution in number theory, an introduction, volume 237 of NATO Sci. Ser. II Math. Phys. Chem., pages 169-196. Springer, Dordrecht, 2007.

[Fra09] Matthias Franz. Convex - a Maple package for convex geometry - version 1.1.3, 2009. 2009.

[Has04] Brendan Hassett. Equations of universal torsors and Cox rings. In Mathematisches Institut, Georg-August-Universität Göttingen : Seminars Summer Term 2004, pages 135-143. Universitätsdrucke Göttingen, Göttingen, 2004.

[LB11] Pierre Le Boudec. Manin's conjecture for a cubic surface with $2 A_{2}+A_{1}$ singularity type. prépublication arXiv:1105.3495, 2011.

[LB12] Pierre Le Boudec. Manin's conjecture for two quartic del Pezzo surfaces with $3 A_{1}$ and $A_{1}+A_{2}$ singularity types. Acta Arith., 151(2):109-163, 2012.

[Lou10] Daniel Loughran. Manin's conjecture for a singular sextic del Pezzo surface. J. Théor. Nombres Bordeaux, 22(3) :675-701, 2010.

[Pey95] Emmanuel Peyre. Hauteurs et mesures de Tamagawa sur les variétés de Fano. Duke Math. J., 79(1) :101-218, 1995.

[Pey03] Emmanuel Peyre. Points de hauteur bornée sur les variétés de drapeaux en caractéristique finie. arXiv:math/0303067v1, 2003.

DAvid Bourqui, IRMAR, Université de Rennes 1, Campus de Beaulieu, 35042 Rennes Cedex, France • E-mail : david.bourqui@univ-rennes1.fr 